Supporting information

\title{
Bacterial Toxicity of Germanium Nanocrystals Induced by Doping with Boron and Phosphorous
}

Bo Zhi ${ }^{\dagger}{ }^{\text {Yueke }}$ Yang, ${ }^{\ddagger}$ Natalie V. Hudson-Smith,${ }^{\dagger}$ Uwe R. Kortshagen, ${ }^{\ddagger}$ Christy L. Haynes ${ }^{*}, \dagger$

Department of Chemistry and Department of Mechanical Engineering, University of Minnesota Twin cities, Minneapolis, Minnesota 55455, United States

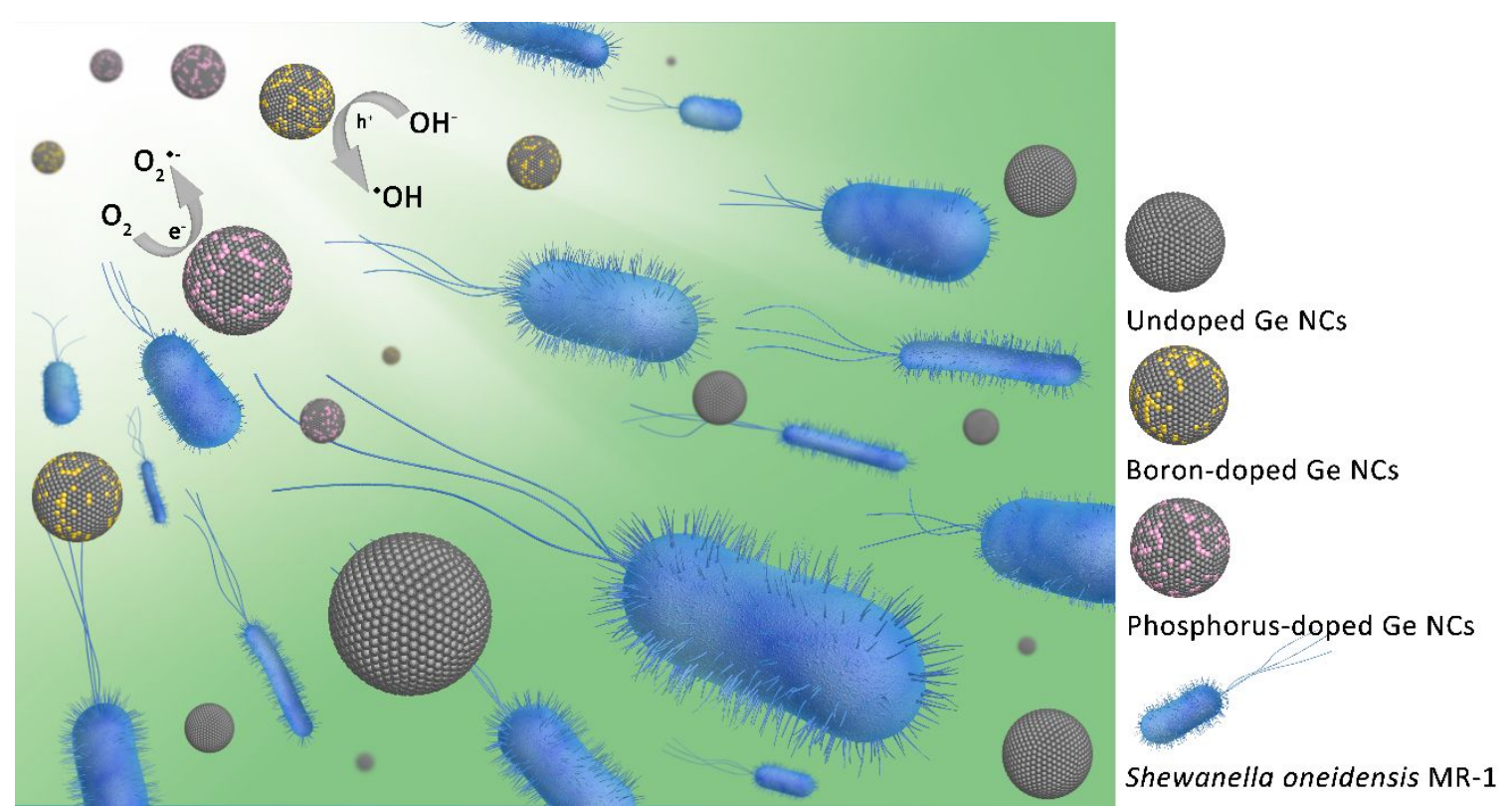

\section{Corresponding Author}

*Dr. Christy L. Haynes: chaynes@umn.edu 


\section{Supporting information list}

Figure S1. Photographic image representing the layout of drop plate colony counting for $S$. oneidensis.

Figure S2. TEM images of 30\% B-doped Ge NCs and 30\% P-doped Ge NCs.

Table S1-5. Lattice constants of Ge NCs.

Figure S3. FTIR spectra for newly synthesized Ge NCs.

Figure S4. High-resolution XPS spectra for doped Ge NCs.

Figure S5. XPS survey spectrum for undoped Ge NCs.

Figure S6. Boron and phosphorus doping levels of Ge NCs evaluated by EDS.

Figure S7. Comparison of doping levels of Ge NCs.

Table S6. Atomic percentage summary of Ge NCs.

Figure S8. DLS results for Ge NCs in either water or HEPES buffer.

Figure S9. Calibration curve to determine the concentration of boron.

Table S7. Boron standards conversion.

Figure S10. Calibration curve to determine the concentration of phosphorus.

Table S8. Phosphorus standards conversion.

Figure S11. Bacterial toxic effects of supernatants of doped Ge NCs.

Figure S12. Enlarged views for the ROS generation of varied Ge NCs.

Figure S13. Hydroxyl radical generation at 15-minute and 1-hour time intervals, as measured by HPF assays.

Figure S14. Superoxide radical generation at 15-minute and 1-hour time intervals, as measured by DHE assays.

Figure S15 through S20. Cytoviva spectral libraries for S. oneidensis cells and Ge NCs.

Figure S21 through S25. Cytoviva enhanced darkfield analysis of the association between $S$. oneidensis cells with undoped Ge NCs, 10\% B-doped Ge NCs, 30\% B-doped Ge NCs, 10\% Pdoped Ge NCs, and 30\% P-doped Ge NCs.

Figure S26. Bright field and dark field TEM images of S. oneidensis cells exposed to $30 \%$ Bdoped Ge NCs and 30\% P-doped Ge NCs. 


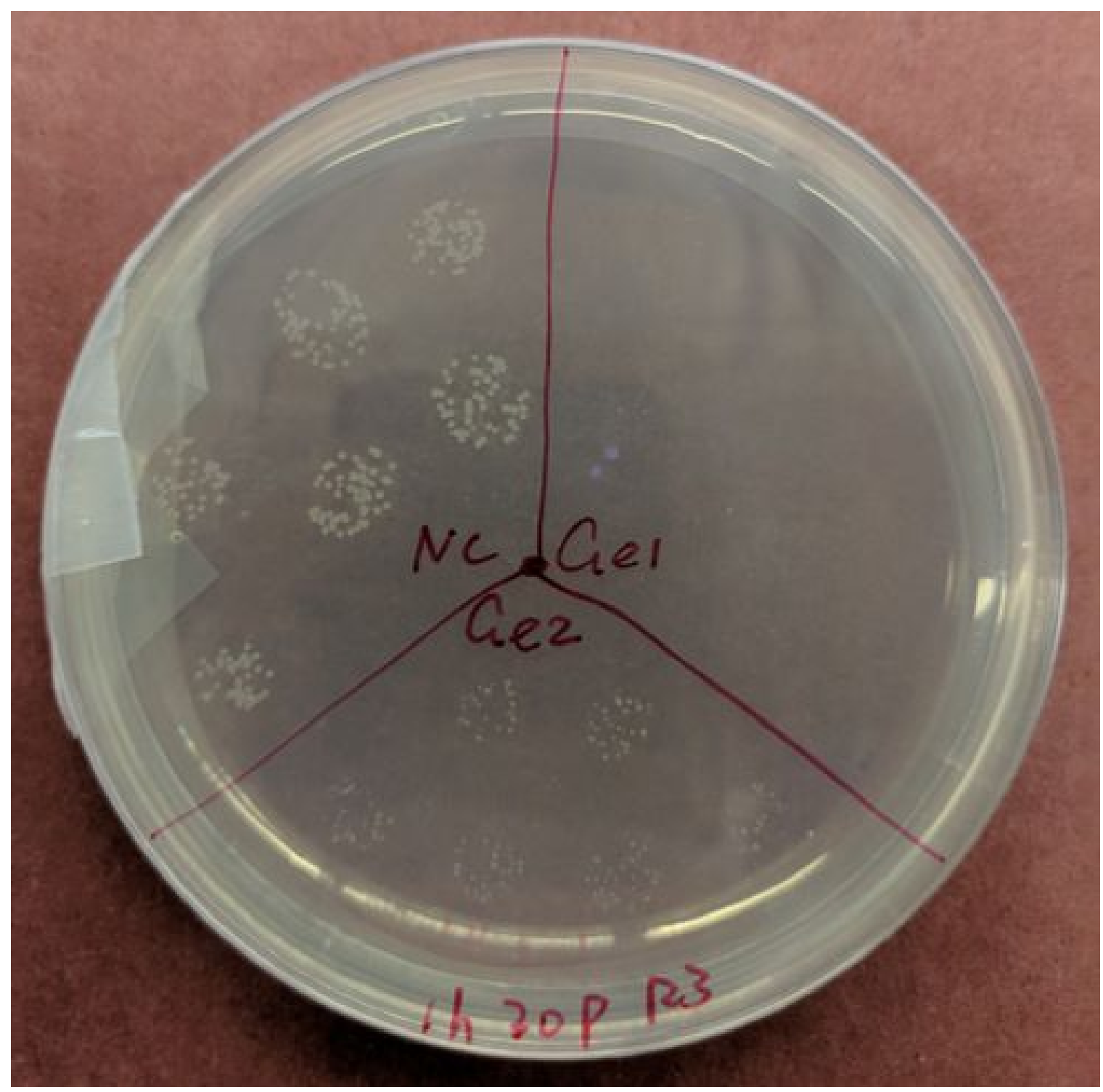

Figure S1. Photographic image representing the layout of drop plate colony counting for $S$. oneidensis. Each small white/gray drop on the plate is a bacterial colony. 

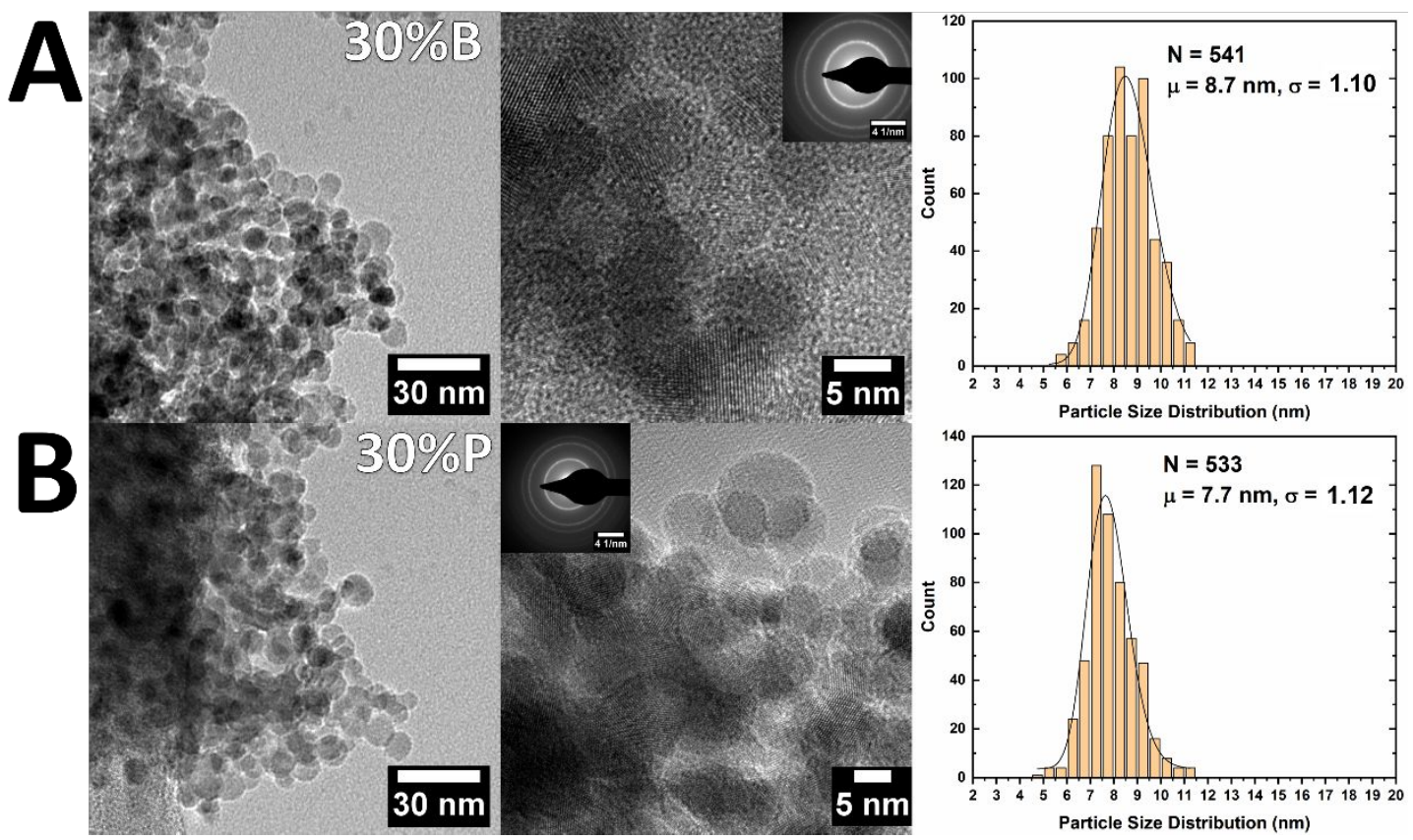

Figure S2. TEM images and size analysis of 30\% B-doped Ge NCs and 30\% P-doped Ge NCs with $\mathrm{N}=500+$ particles counted. 
Table S1. $d$-spacing values of undoped Ge NCs (calculated using the SAED pattern).

\begin{tabular}{ccc}
\hline Ring radius $\left(\mathbf{n m}^{-1}\right)$ & $\boldsymbol{d}$-spacing $(\mathbf{n m})$ & $(\boldsymbol{h k l})$ \\
\hline $\mathbf{3 . 0 2 1}$ & $\mathbf{0 . 3 3 1}$ & $(111)$ \\
$\mathbf{5 . 1 2 4}$ & 0.195 & $(220)$ \\
$\mathbf{5 . 9 8 9}$ & 0.167 & $(311)$ \\
\hline
\end{tabular}

Table S2. $d$-spacing values of $10 \%$ B-doped Ge NCs (calculated using the SAED pattern).

\begin{tabular}{ccc}
\hline Ring radius $\left(\mathbf{n m}^{-1}\right)$ & $\boldsymbol{d}$-spacing $(\mathbf{n m})$ & $(\boldsymbol{h k l})$ \\
\hline $\mathbf{3 . 1 3 5}$ & 0.319 & $(111)$ \\
$\mathbf{5 . 2 9 1}$ & 0.189 & $(220)$ \\
$\mathbf{6 . 2 1 1}$ & 0.161 & $(311)$ \\
\hline
\end{tabular}

Table S3. $d$-spacing values of 30\% B-doped Ge NCs (calculated using the SAED pattern).

\begin{tabular}{ccc}
\hline Ring radius $\left(\mathbf{n m}^{-1}\right)$ & $\boldsymbol{d}$-spacing $(\mathbf{n m})$ & $($ hkl $)$ \\
\hline $\mathbf{3 . 0 3 1}$ & 0.330 & $(111)$ \\
$\mathbf{5 . 0 7 6}$ & 0.197 & $(220)$ \\
$\mathbf{5 . 7 8 0}$ & 0.173 & $(311)$
\end{tabular}

Table S4. $d$-spacing values of $10 \%$ P-doped Ge NCs (calculated using the SAED pattern).

\begin{tabular}{ccc}
\hline Ring radius $\left(\mathbf{n m}^{-1}\right)$ & $\boldsymbol{d}$-spacing $(\mathbf{n m})$ & $($ hkl $)$ \\
\hline $\mathbf{3 . 0 4 9}$ & 0.328 & $(111)$ \\
$\mathbf{4 . 9 2 6}$ & 0.203 & $(220)$ \\
$\mathbf{5 . 7 4 7}$ & 0.174 & $(311)$ \\
\hline
\end{tabular}

Table S5. $d$-spacing values of 30\% P-doped Ge NCs (calculated using the SAED pattern).

\begin{tabular}{ccc}
\hline Ring radius $\left(\mathbf{n m}^{-1}\right)$ & $\boldsymbol{d}$-spacing $(\mathbf{n m})$ & $(\boldsymbol{h k l})$ \\
\hline $\mathbf{3 . 0 7 7}$ & 0.325 & $(111)$ \\
$\mathbf{5 . 4 6 4}$ & 0.183 & $(220)$ \\
$\mathbf{5 . 8 8 3}$ & 0.170 & $(311)$ \\
\hline
\end{tabular}




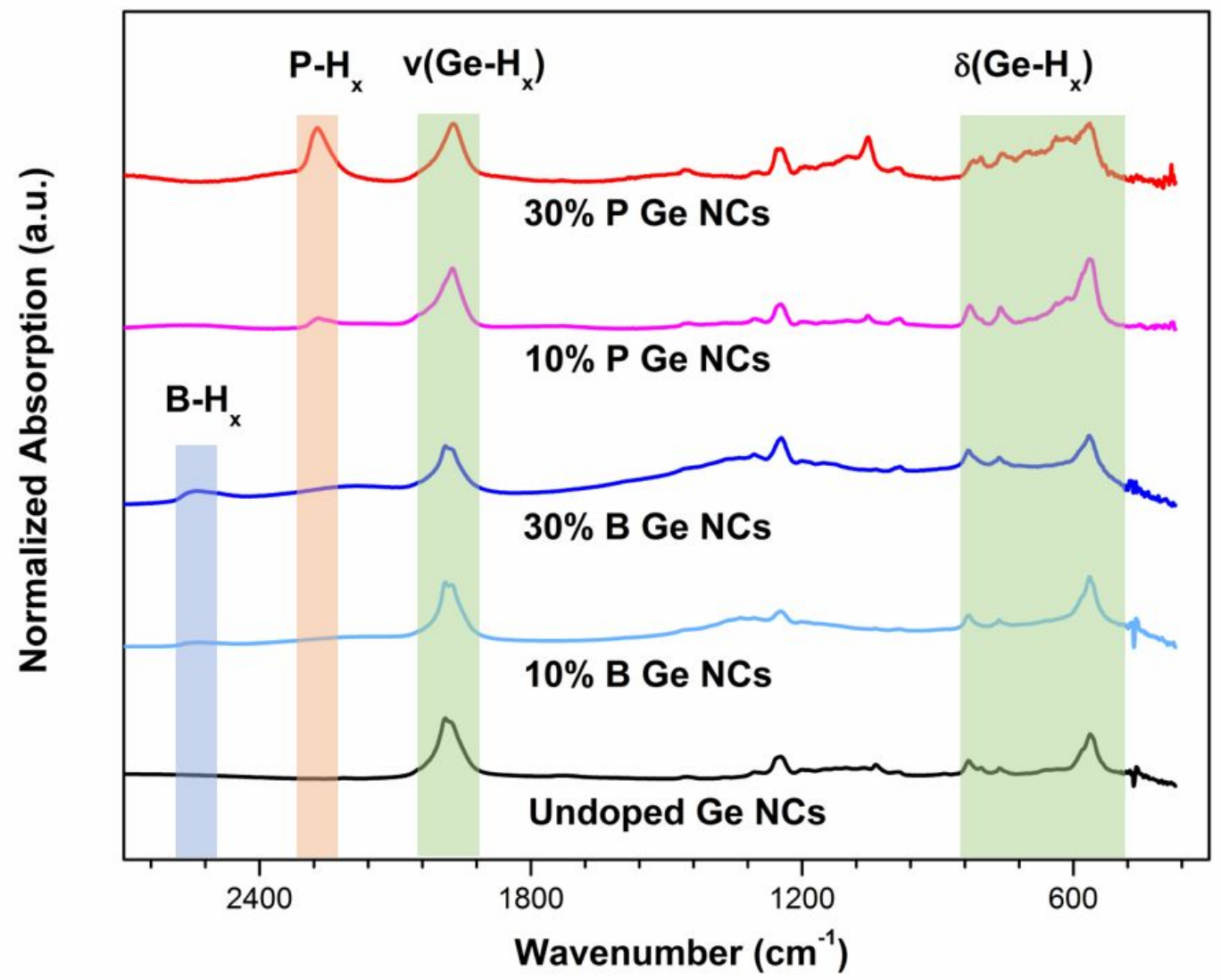

Figure S3. FTIR spectra for freshly synthesized Ge NCs. 

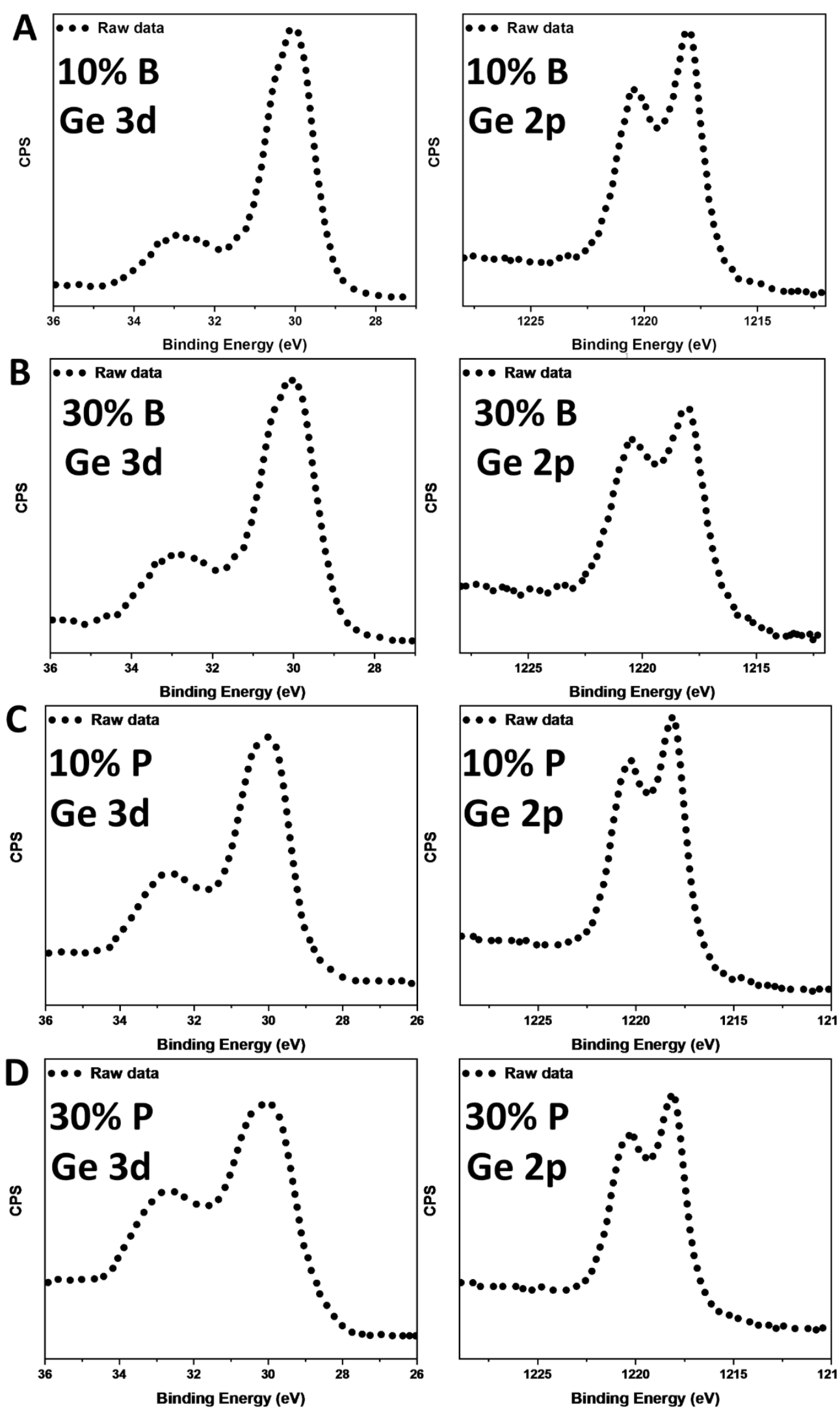

Figure S4. High-resolution XPS spectra for doped Ge NCs. 


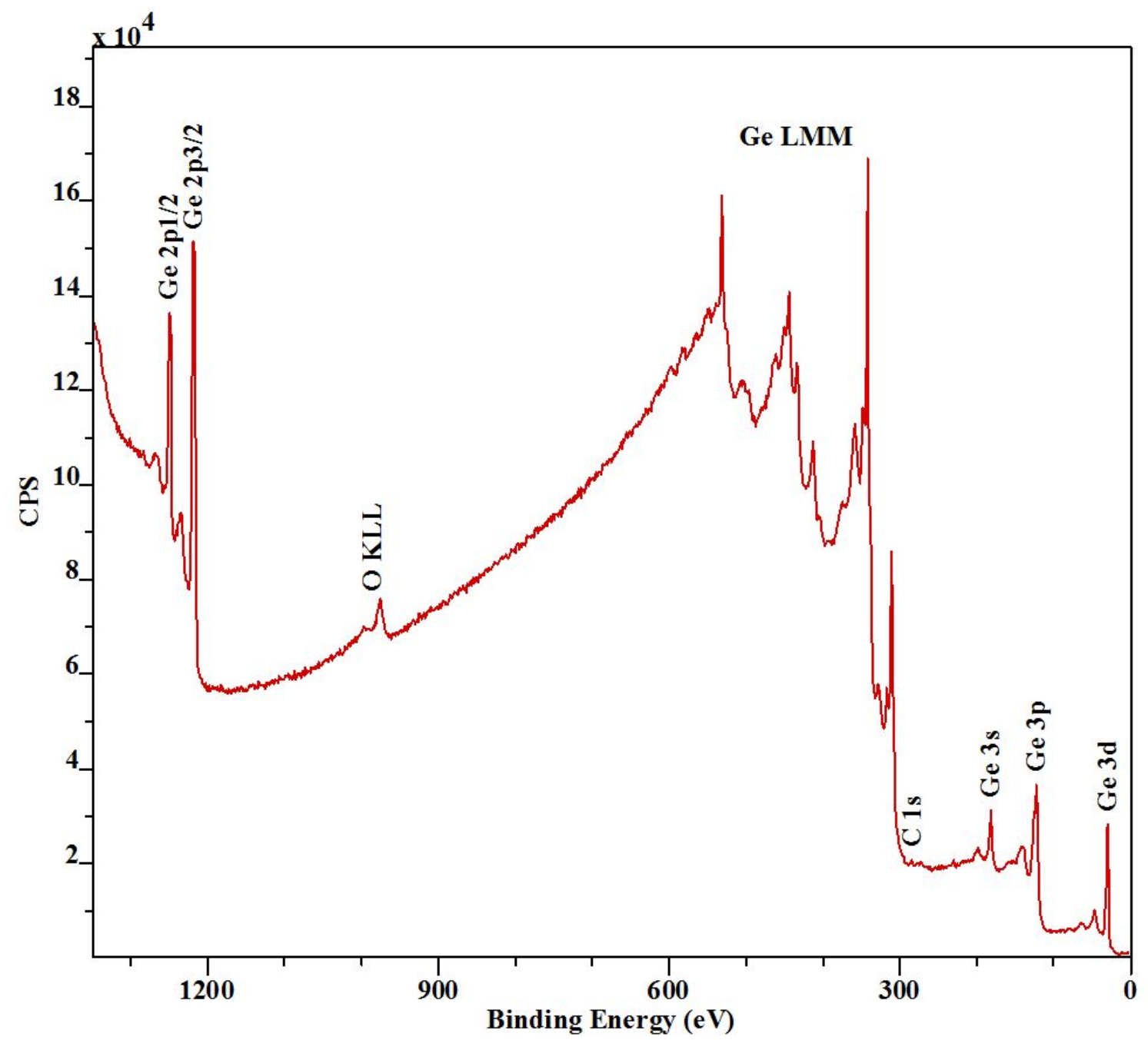

Figure S5. XPS survey spectrum for undoped Ge NCs.

It is challenging to assess the atomic percentage of oxygen, boron, and phosphorus because $\mathrm{Ge}$ LMM peak groups (300-600 eV) overlap with O1s (531 eV).

Similarly, for doped Ge NCs, it is also difficult to gauge boron and phosphorus incorporation because Ge3s (180 eV) overlaps with B1s $(188 \mathrm{eV})$ and P2s $(189 \mathrm{eV})$ while Ge 3p (120-125 eV) overlaps with P2p (135-136 eV). 


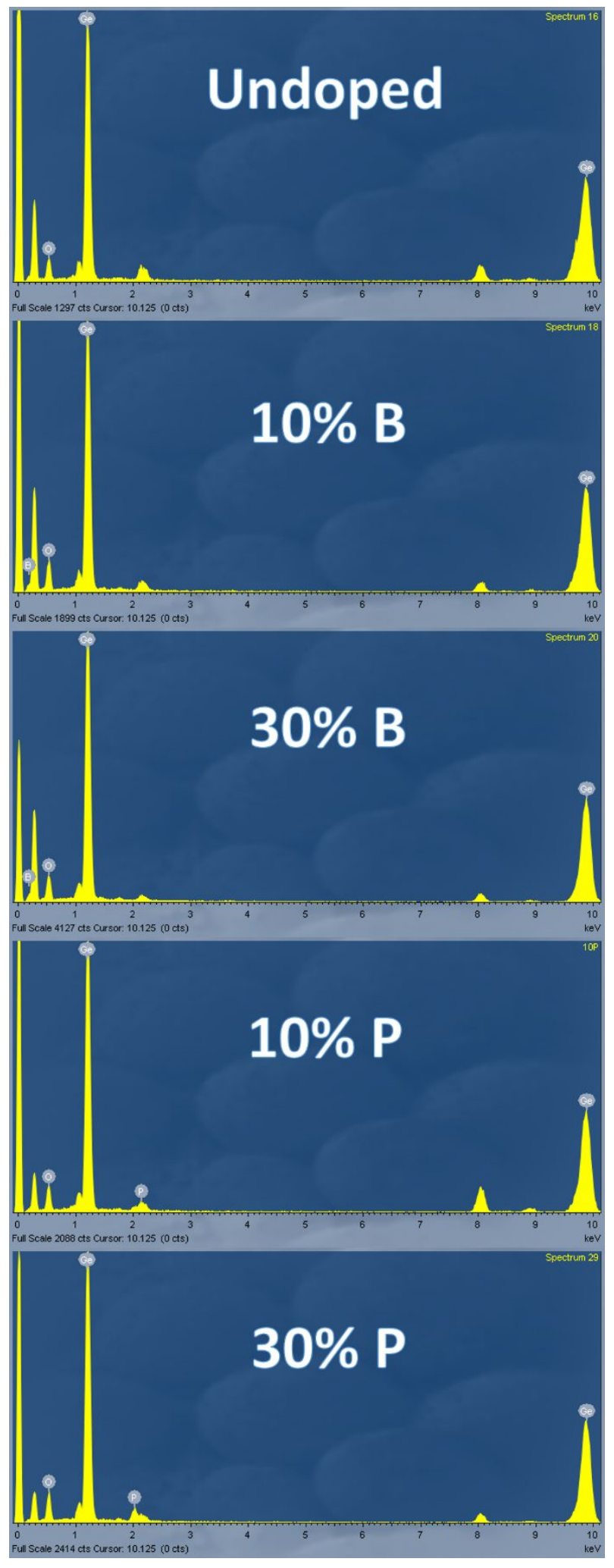

Figure S6. Boron and phosphorus doping levels of Ge NCs evaluated by EDS (Energy dispersive $\mathrm{x}$-ray spectroscopy). 


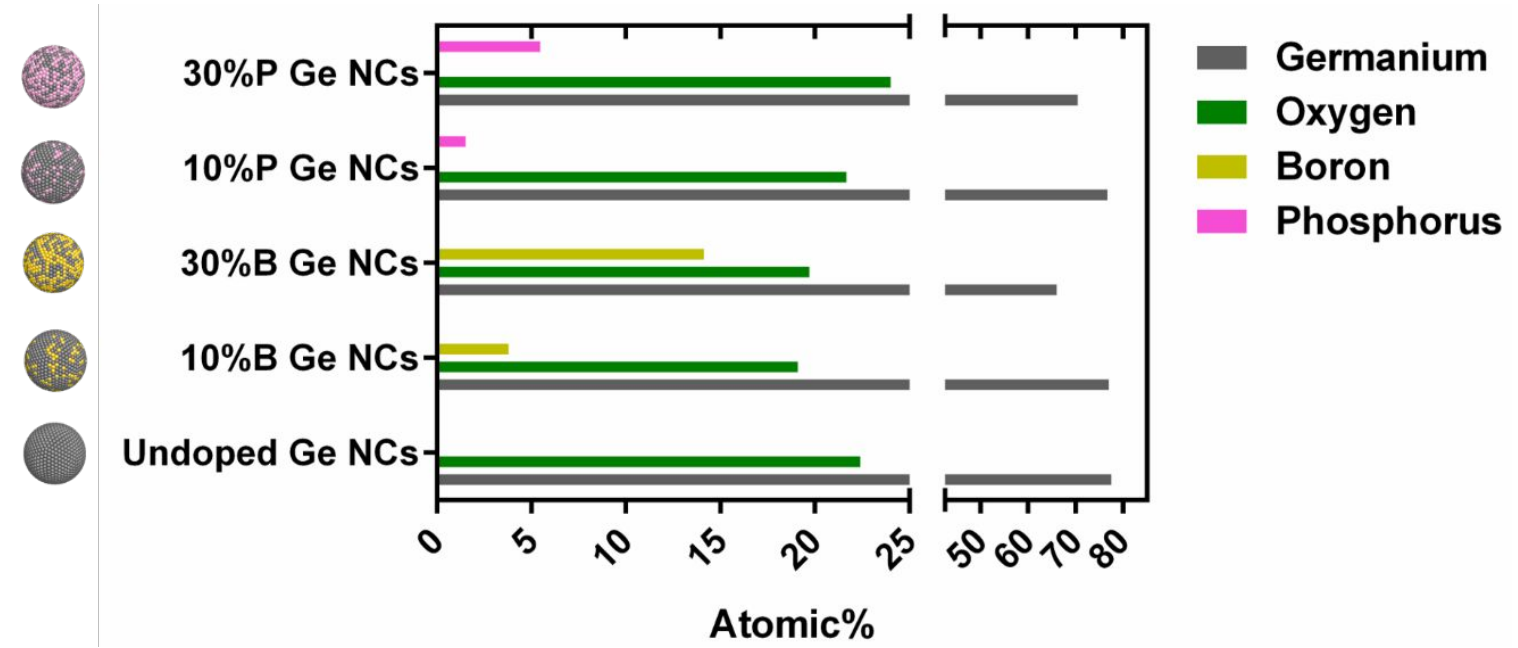

Figure S7. Comparison of doping levels of Ge NCs.

Table S6. Atomic percentage summary of Ge NCs.

\begin{tabular}{ccccc}
\hline Atomic\% (N $=4$ or 5) & Germanium & Oxygen & Boron & Phosphorus \\
\hline Undoped Ge NCs & $77.6 \pm 1.1$ & $22.4 \pm 1.1$ & N/A & N/A \\
$10 \% \mathrm{~B}$ Ge NCs & $77.1 \pm 3.9$ & $19.1 \pm 3.7$ & $3.8 \pm 0.7$ & N/A \\
$30 \% \mathrm{~B}$ Ge NCs & $66.1 \pm 1.8$ & $19.7 \pm 1.8$ & $14.1 \pm 1.9$ & N/A \\
$10 \% \mathrm{P} \mathrm{Ge} \mathrm{NCs}$ & $76.8 \pm 0.7$ & $21.7 \pm 0.6$ & N/A & $1.5 \pm 0.1$ \\
$30 \% \mathrm{P} \mathrm{Ge} \mathrm{NCs}$ & $70.5 \pm 0.3$ & $24.0 \pm 0.5$ & N/A & $5.5 \pm 0.2$ \\
\hline
\end{tabular}



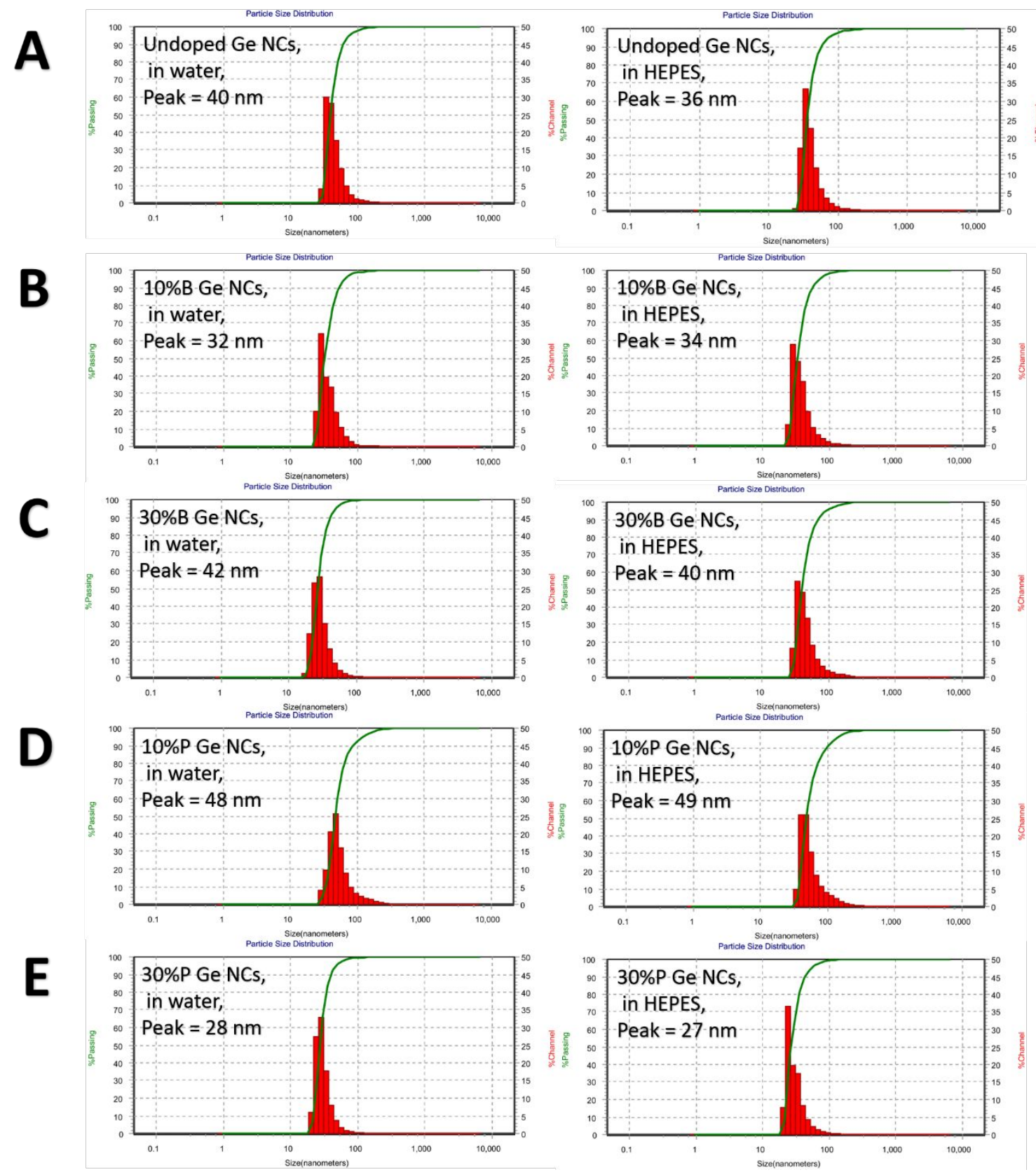

Figure S8. Dynamic light scattering (DLS) results for Ge NCs in both water (left) or HEPES buffer (right). 


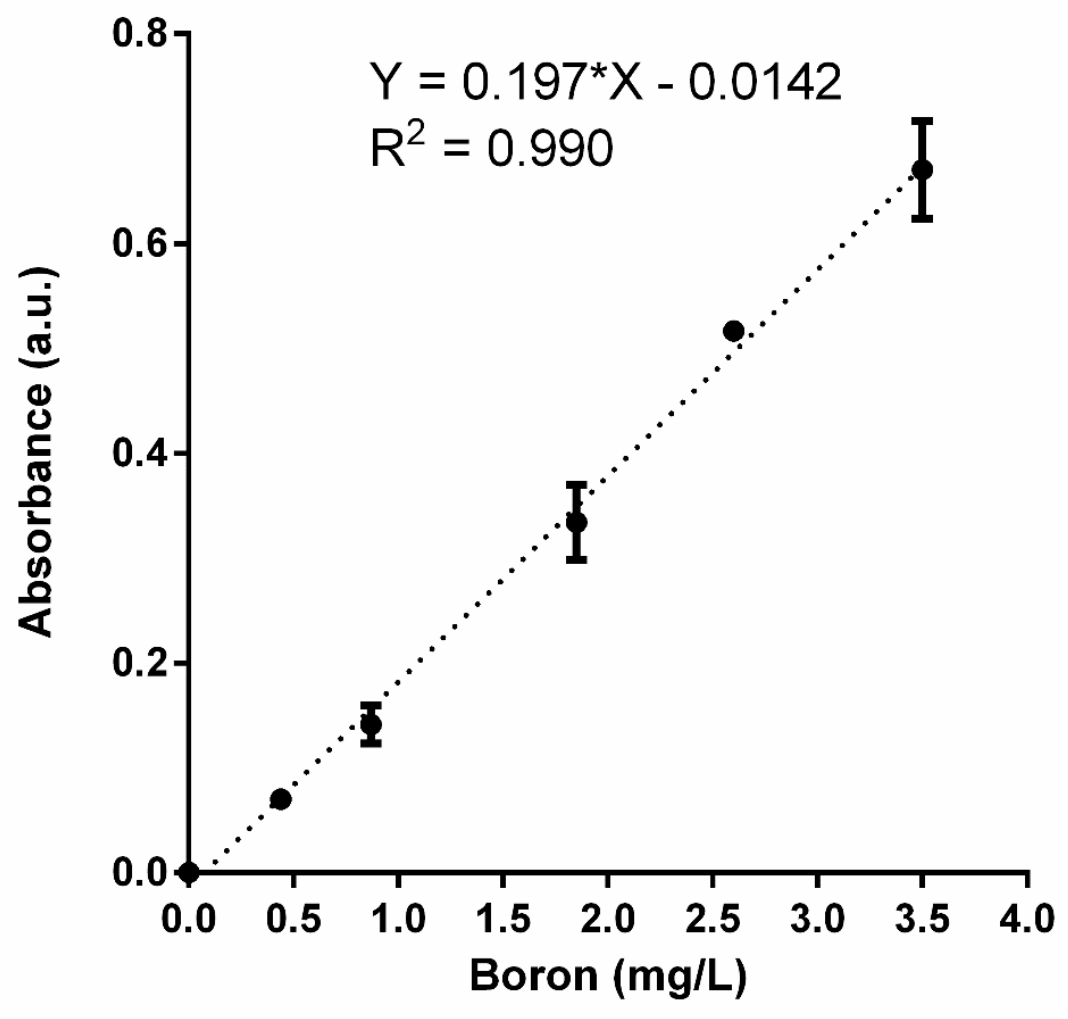

Figure S9. Calibration curve to determine the concentration of boron. Curcumin reacts with borates to form red colored rosocyanine, which absorbs at $540 \mathrm{~nm} .(\mathrm{n}=3)$

Table S7. Boron standards conversion.

\begin{tabular}{cc}
\hline Boric acid $\mathrm{mg} / \mathrm{L}$ & Boron $\mathrm{mg} / \mathrm{L}$ \\
\hline 0 & 0 \\
2.5 & 0.4 \\
5.0 & 0.9 \\
10.0 & 1.85 \\
15.0 & 2.6 \\
20.0 & 3.5 \\
\hline
\end{tabular}

Based on the calibration equation ( $\mathrm{Y}=0.197 * \mathrm{X}-0.0142)$, it can be back-calculated that for $10 \%$ B-doped Ge NCs $(1000 \mathrm{mg} / \mathrm{L})$, the supernatant boron concentration is $1.5 \pm 0.2 \mathrm{mg} / \mathrm{L}$ while for $30 \%$ B-doped Ge NCs $(1000 \mathrm{mg} / \mathrm{L})$, it is $3.5 \pm 0.1 \mathrm{mg} / \mathrm{L}$. 


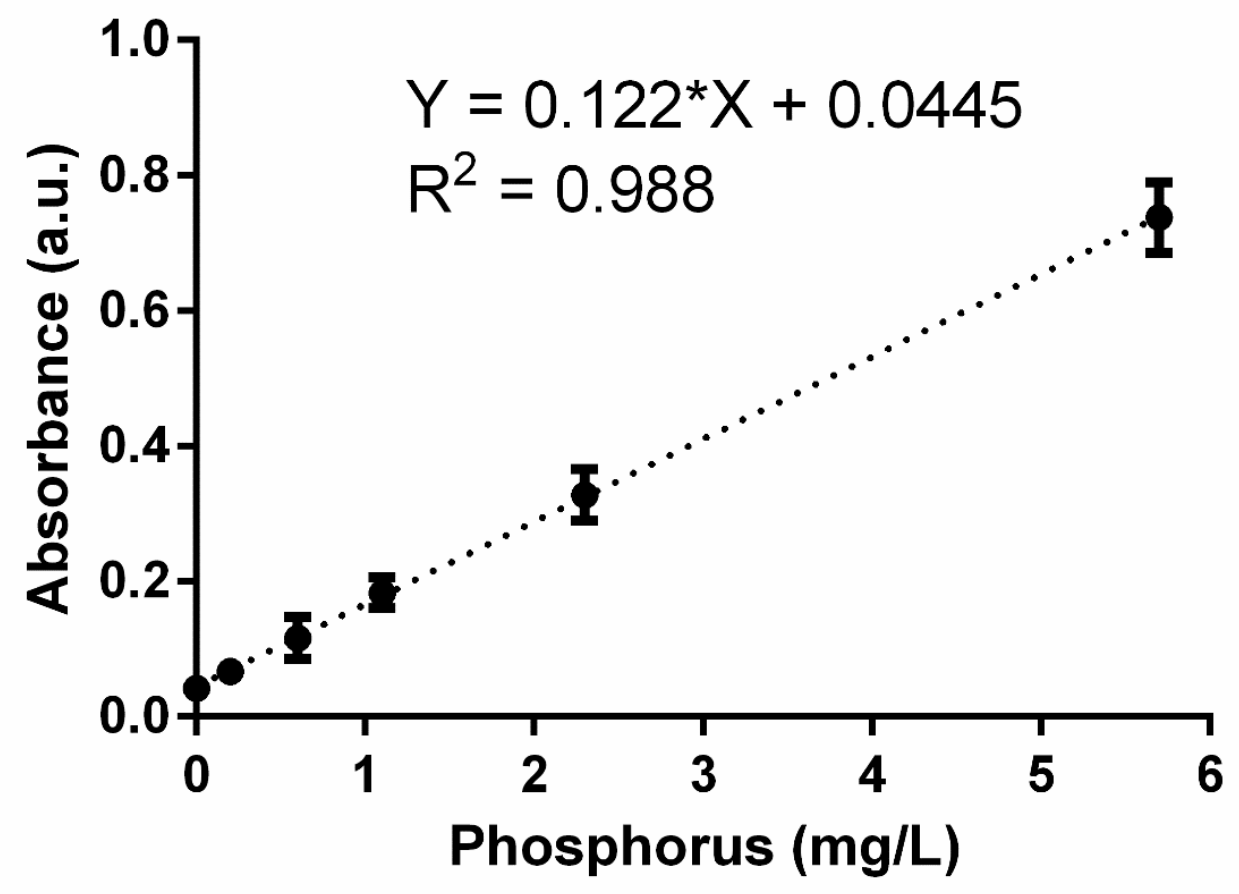

Figure S10. Calibration curve to determine the concentration of phosphorus. Antimony potassium tartrate and ammonium molybdate react with orthophosphate to form an antimony-phosphomolybdate complex, which can be reduced to a blue complex by ascorbic acid. Its absorbance is measured at $670 \mathrm{~nm} .(\mathrm{n}=3)$

Table S8. Phosphorus standards conversion

\begin{tabular}{cc}
\hline $\mathrm{KH}_{2} \mathrm{PO}_{4} \mathrm{mg} / \mathrm{L}$ & Phosphorus mg/L \\
\hline 0 & 0 \\
1 & 0.2 \\
2.5 & 0.6 \\
5 & 1.1 \\
10 & 2.3 \\
25 & 5.7 \\
\hline
\end{tabular}

Based on the calibration equation ( $\mathrm{Y}=0.122 * \mathrm{X}+0.0445)$, it can be back-calculated that for $10 \%$ P-doped Ge NCs $(1000 \mathrm{mg} / \mathrm{L})$, the supernatant phosphorus concentration is $1.8 \pm 0.1 \mathrm{mg} / \mathrm{L}$ while for $30 \%$ P-doped Ge NCs $(1000 \mathrm{mg} / \mathrm{L})$, it is $2.3 \pm 0.1 \mathrm{mg} / \mathrm{L}$. 
Supernatants of doped Ge NCs: $1000 \mathrm{mg} / \mathrm{L}, 15-\mathrm{minute}$ exposure

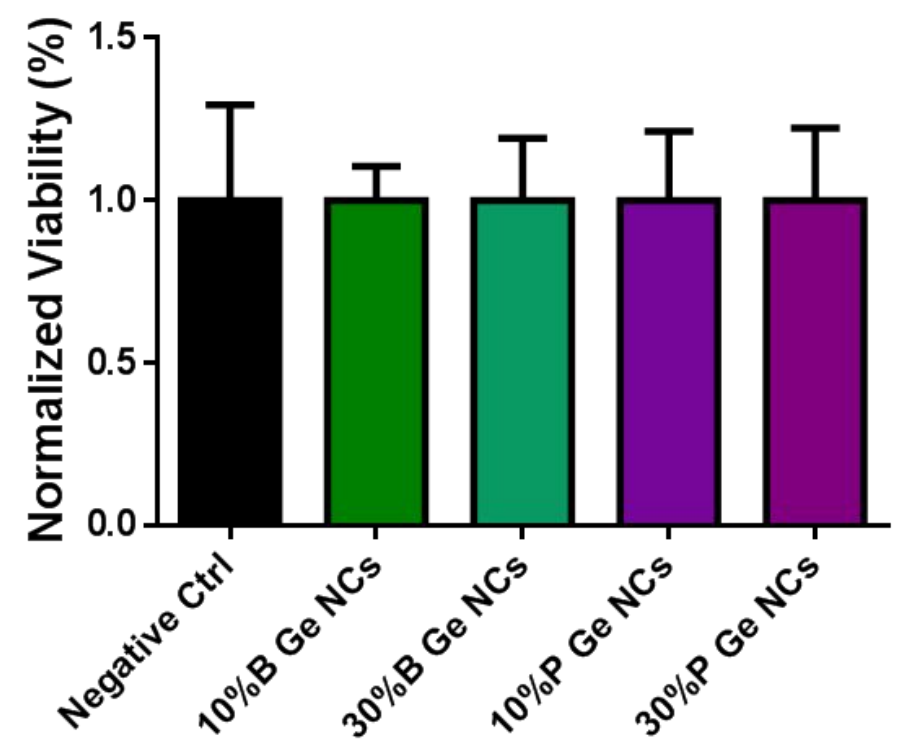

Supernatants of doped Ge NCs: $1000 \mathrm{mg} / \mathrm{L}$, 1-hour exposure

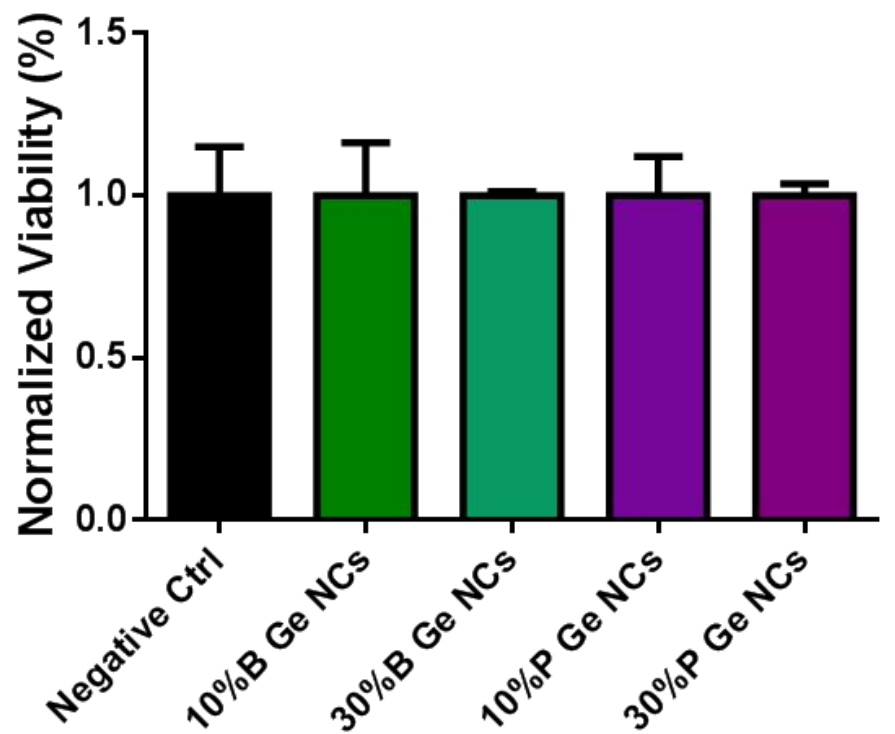

Figure S11. Colony counting experiments using the supernatants of Ge NCs. There are no significant differences among the different experimental conditions. 

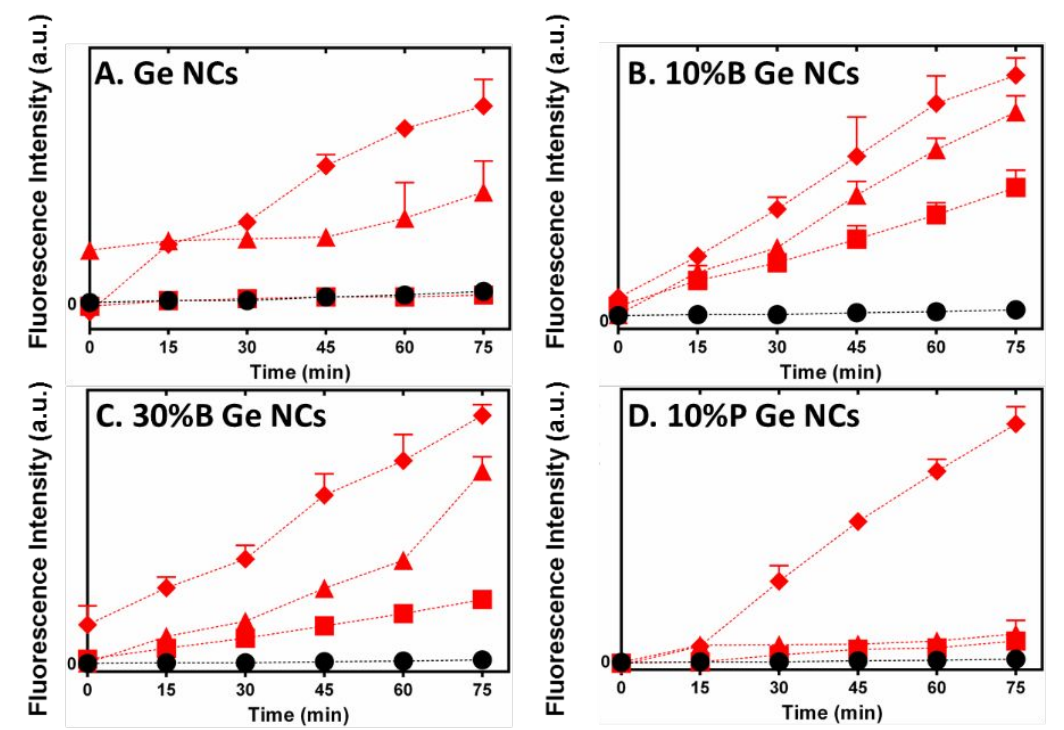
$-100 \mathrm{mg} / \mathrm{L}$ of $\mathrm{NCs}$
$-1000 \mathrm{mq} / \mathrm{L}$ of $\mathrm{NCs}$

Negative Ctrl $10 \mathrm{mg} / \mathrm{L}$ of $\mathrm{NCs}$

Figure S12. Enlarged views for the abiotic ROS generation of varied Ge NCs, determined by the DCFDA assay (incubation time $1.25 \mathrm{~h}, \mathrm{n}=3$ ). 


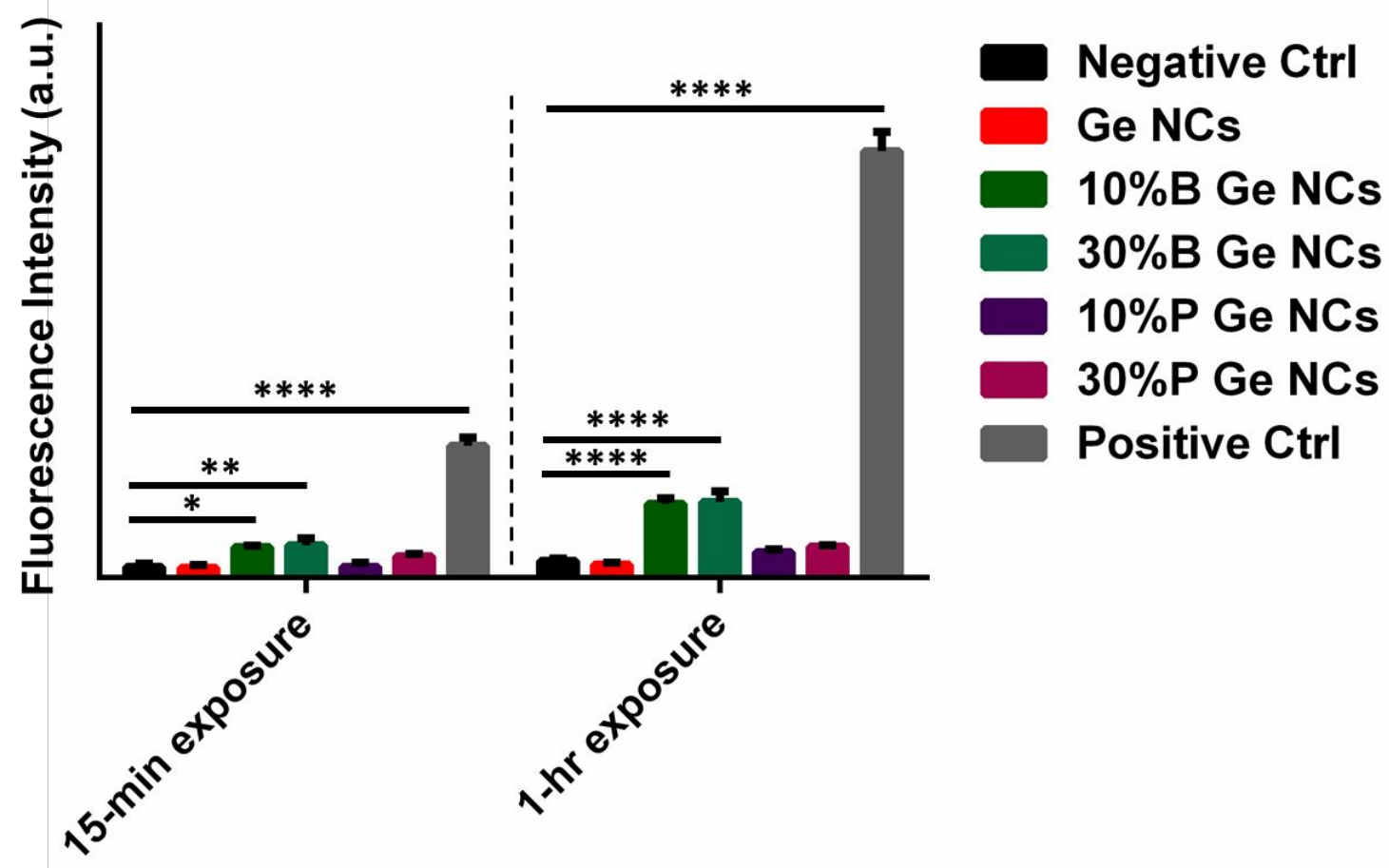

Figure S13. Hydroxyl radical generation at 15-minute and 1-hour time intervals, as measured by cell-free HPF assays. The asterisks indicate statistical significance level: ${ }^{*} \mathrm{p}<0.05,{ }^{*} \mathrm{p}<0.01$, $* * * p<0.001, * * * * p<0.0001$ (examined by two-way ANOVA, followed by Sidak's multiple comparison tests, $\mathrm{n}=3$ ). 


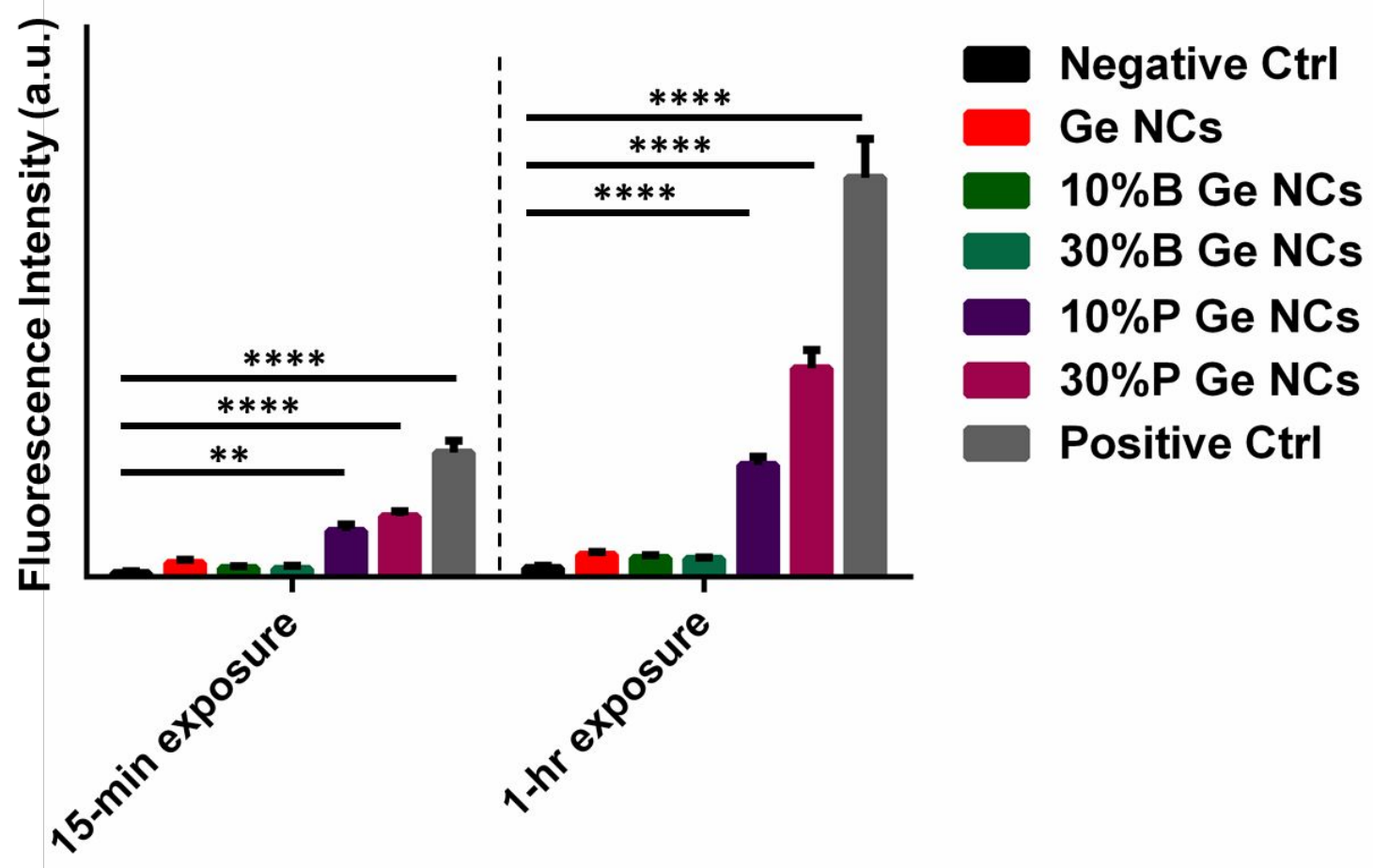

Figure S14. Superoxide radical generation at 15-minute and 1-hour time intervals, as measured by DHE assays. The asterisks indicate statistical significance level: ${ }^{*} \mathrm{p}<0.05,{ }^{*} \mathrm{p}<0.01,{ }^{* * *} \mathrm{p}<0.001$, $* * * * \mathrm{p}<0.0001$ (examined by two-way ANOVA, followed by Sidak's multiple comparison tests, $\mathrm{n}$ $=3$ ). 

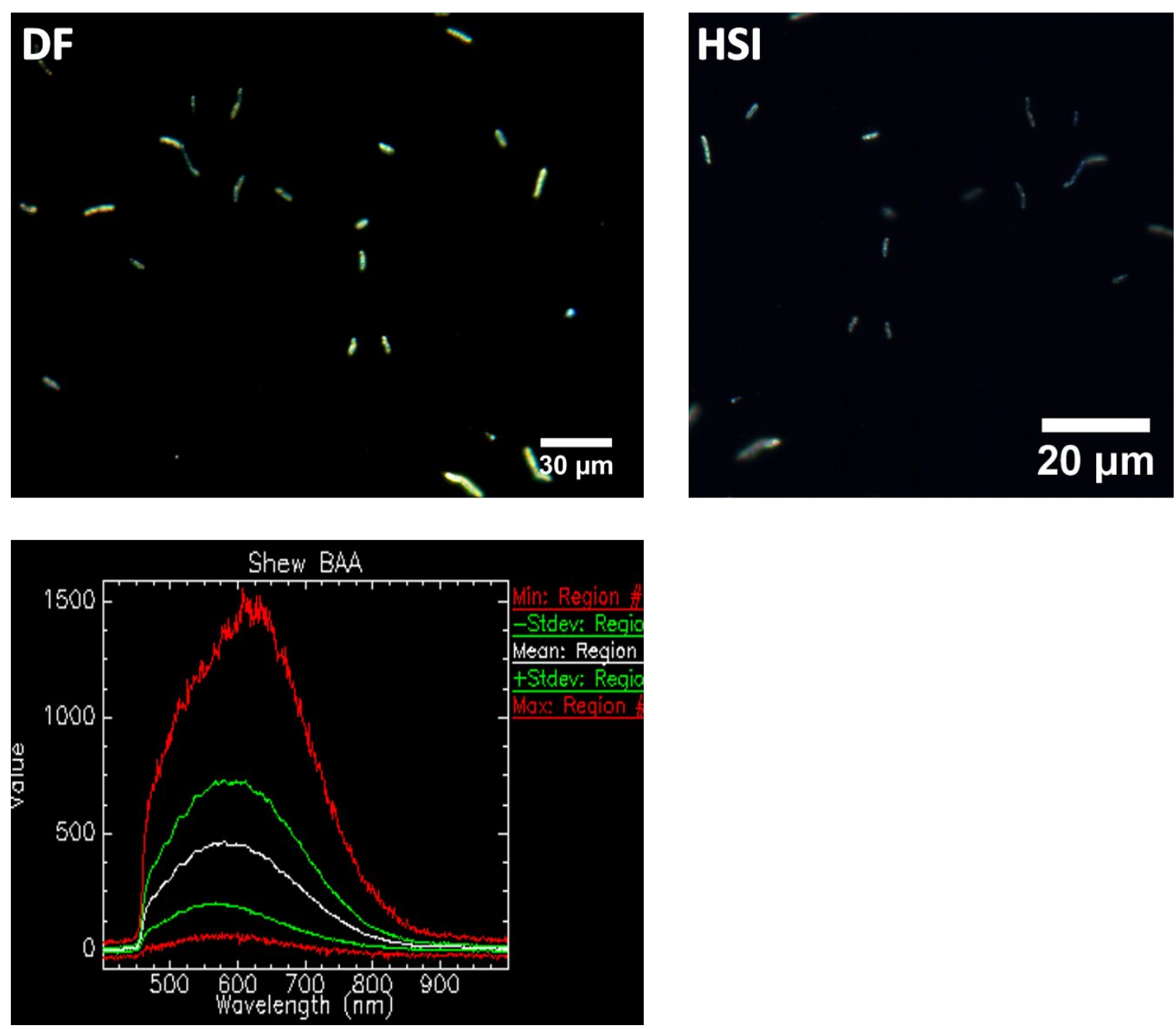

Figure S15. Cytoviva analysis of S. oneidensis cells: dark-field (DF) image, hyperspectral (HSI) image, and spectral library for $S$. oneidensis cells, generated using the region-of-interest (ROI) tool. 

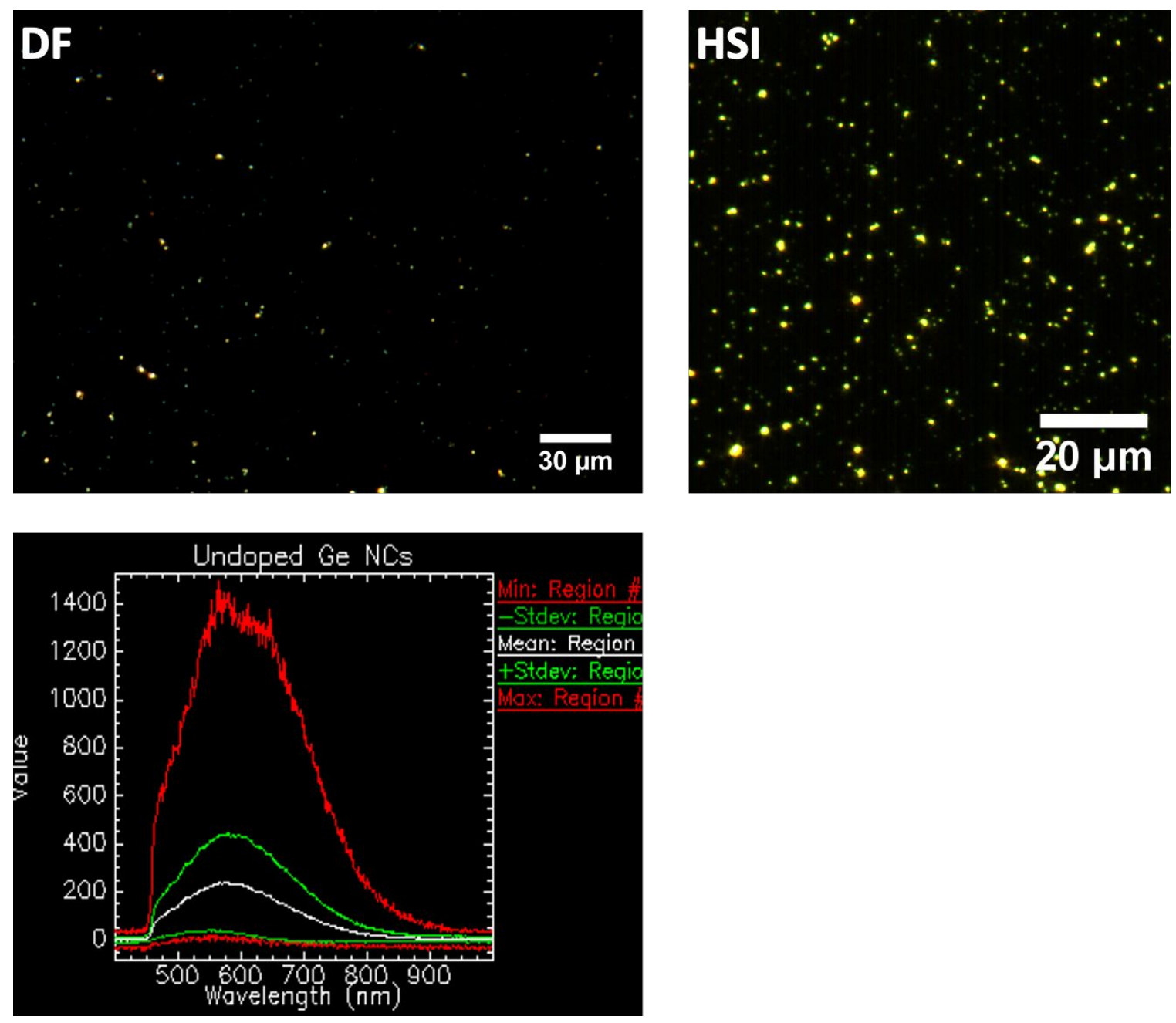

Figure S16. Cytoviva analysis of undoped Ge NCs: dark-field (DF) image, hyperspectral (HSI) image, and spectral library for undoped Ge NCs, generated using the ROI tool. 

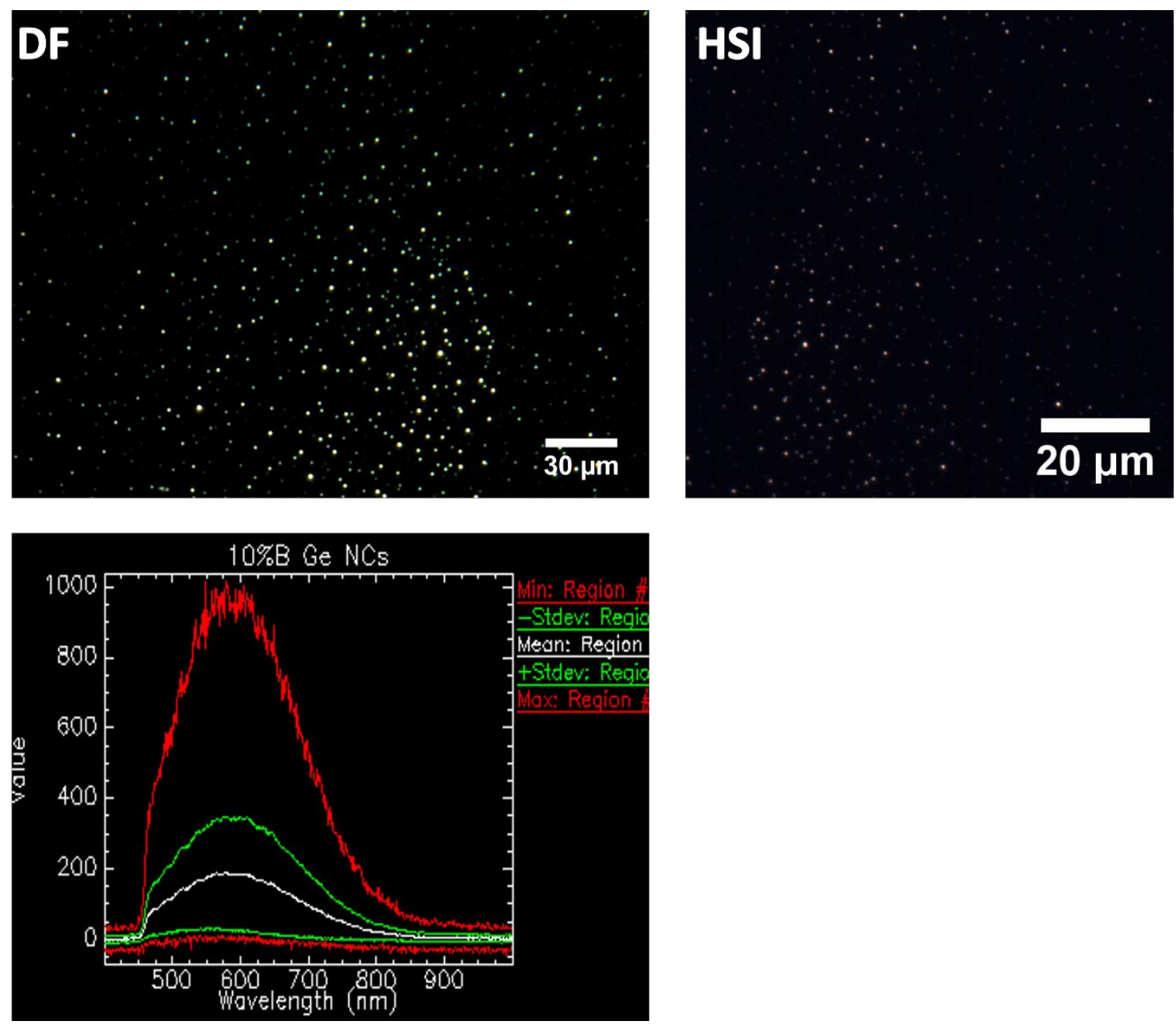

Figure S17. Cytoviva analysis of 10\% B-doped Ge NCs: dark-field (DF) image, hyperspectral (HSI) image, and spectral library for $10 \%$ B-doped Ge NCs, generated using the ROI tool. 

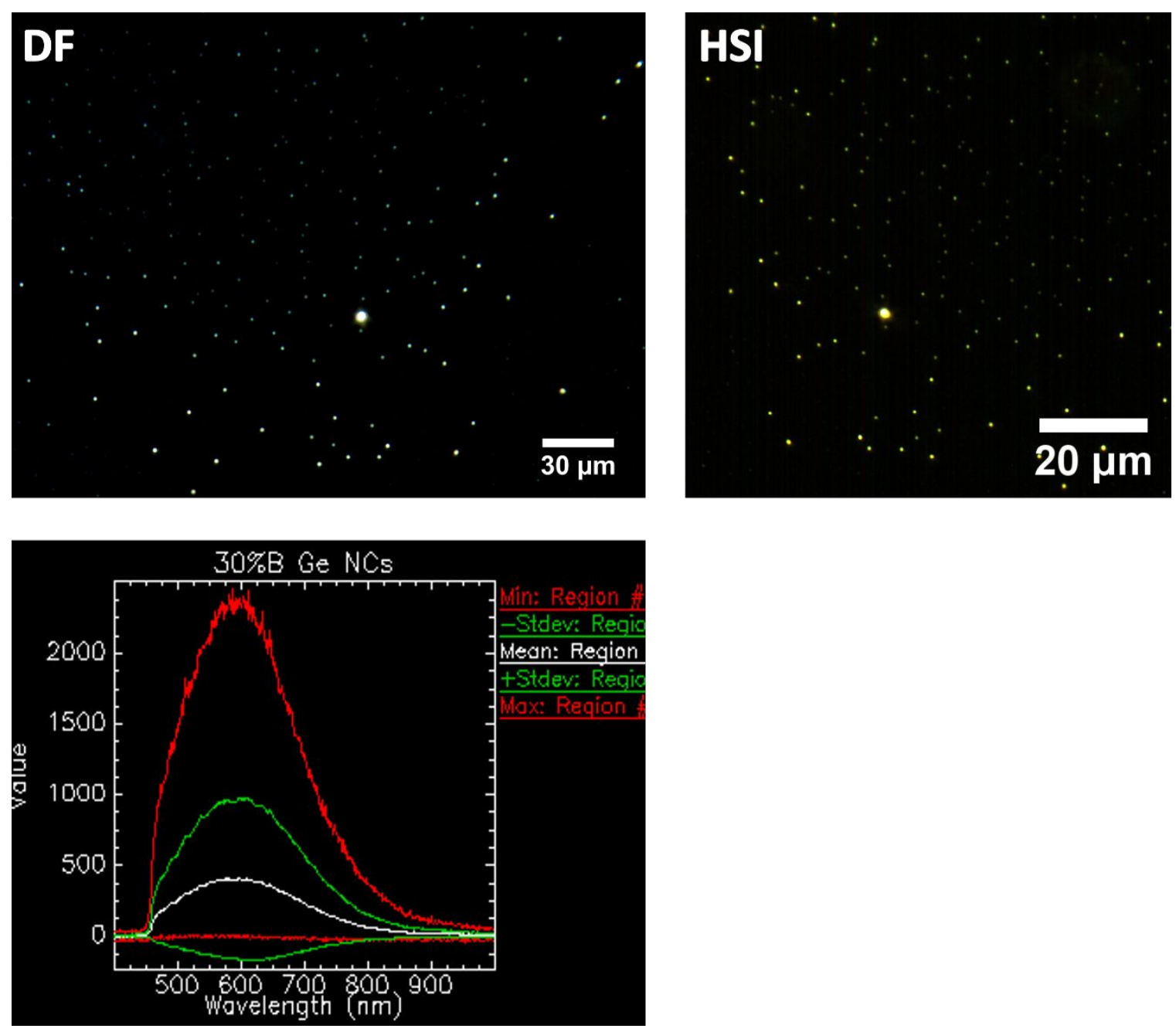

Figure S18. Cytoviva analysis of 30\% B-doped Ge NCs: dark-field (DF) image, hyperspectral (HSI) image, and spectral library for 30\% B-doped Ge NCs, generated using the ROI tool. 

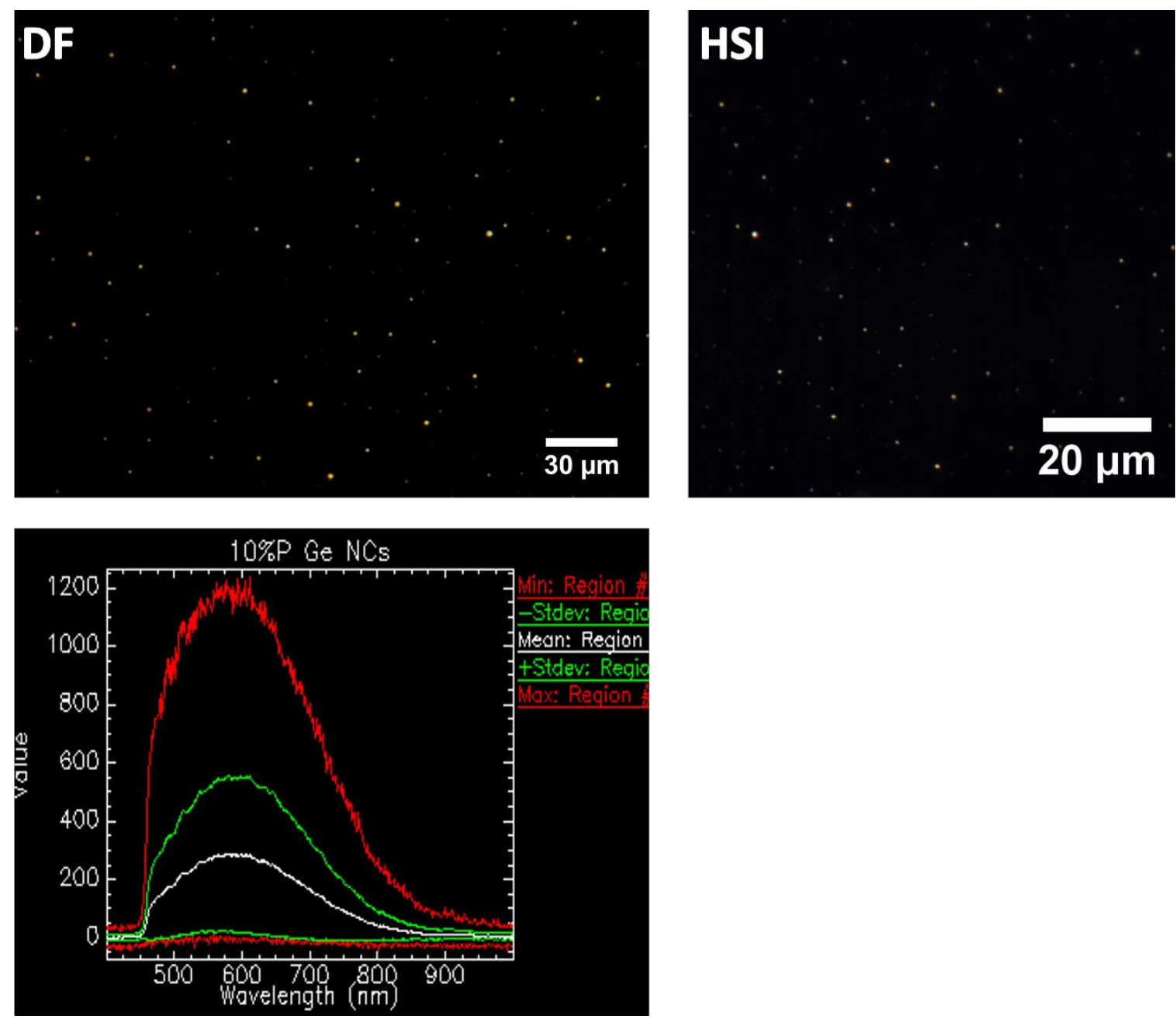

Figure S19. Cytoviva analysis of 10\% P-doped Ge NCs: dark-field (DF) image, hyperspectral (HSI) image, and spectral library for $10 \%$ P-doped Ge NCs, generated using the ROI tool. 

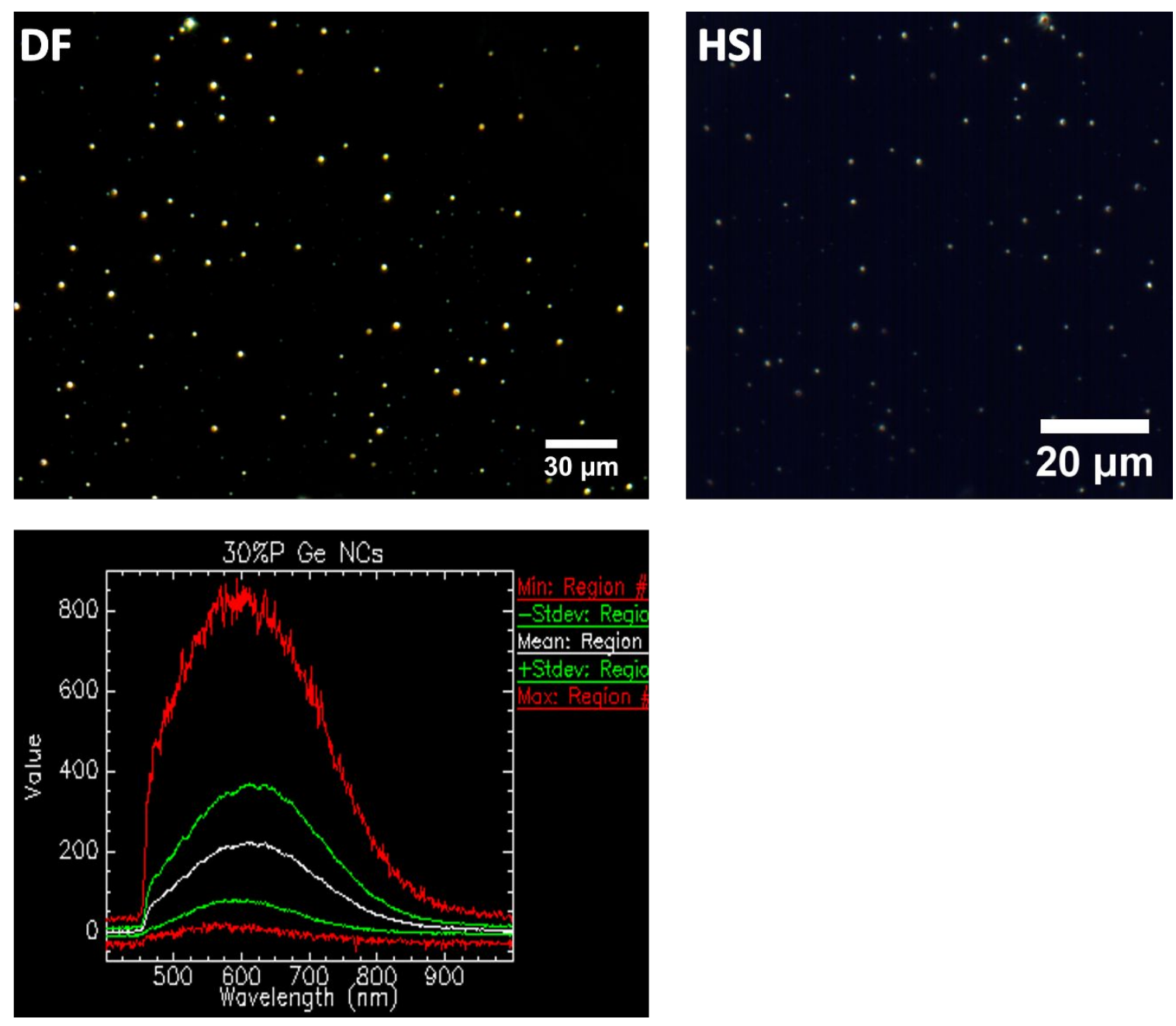

Figure S20. Cytoviva analysis of 30\% P-doped Ge NCs: dark-field (DF) image, hyperspectral (HSI) image, and spectral library for $30 \%$ P-doped Ge NCs, generated using the ROI tool. 


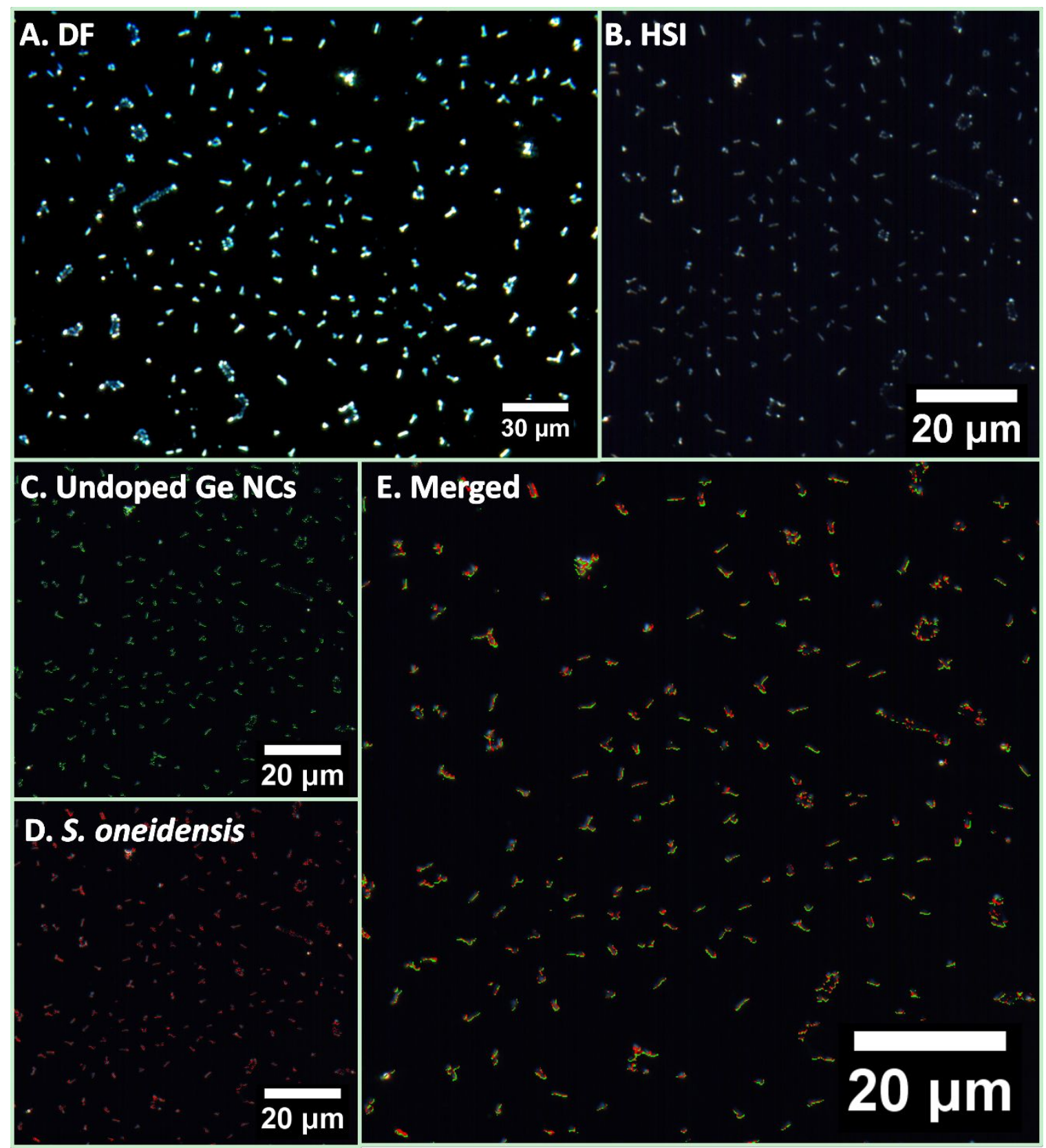

Figure S21. Enhanced dark-field analysis of the association between S. oneidensis cells with undoped Ge NCs: (A) dark-field image of bacteria exposure solution, (B) hyperspectral image of region of interest (ROI) in A. Mapping (C) undoped Ge NCs alone and (D) S. oneidensis cells alone in the hyperspectral data. (E) Merged mapping results of (C) and (D) where bacteria cells are false-colored red while undoped Ge NCs with green. 


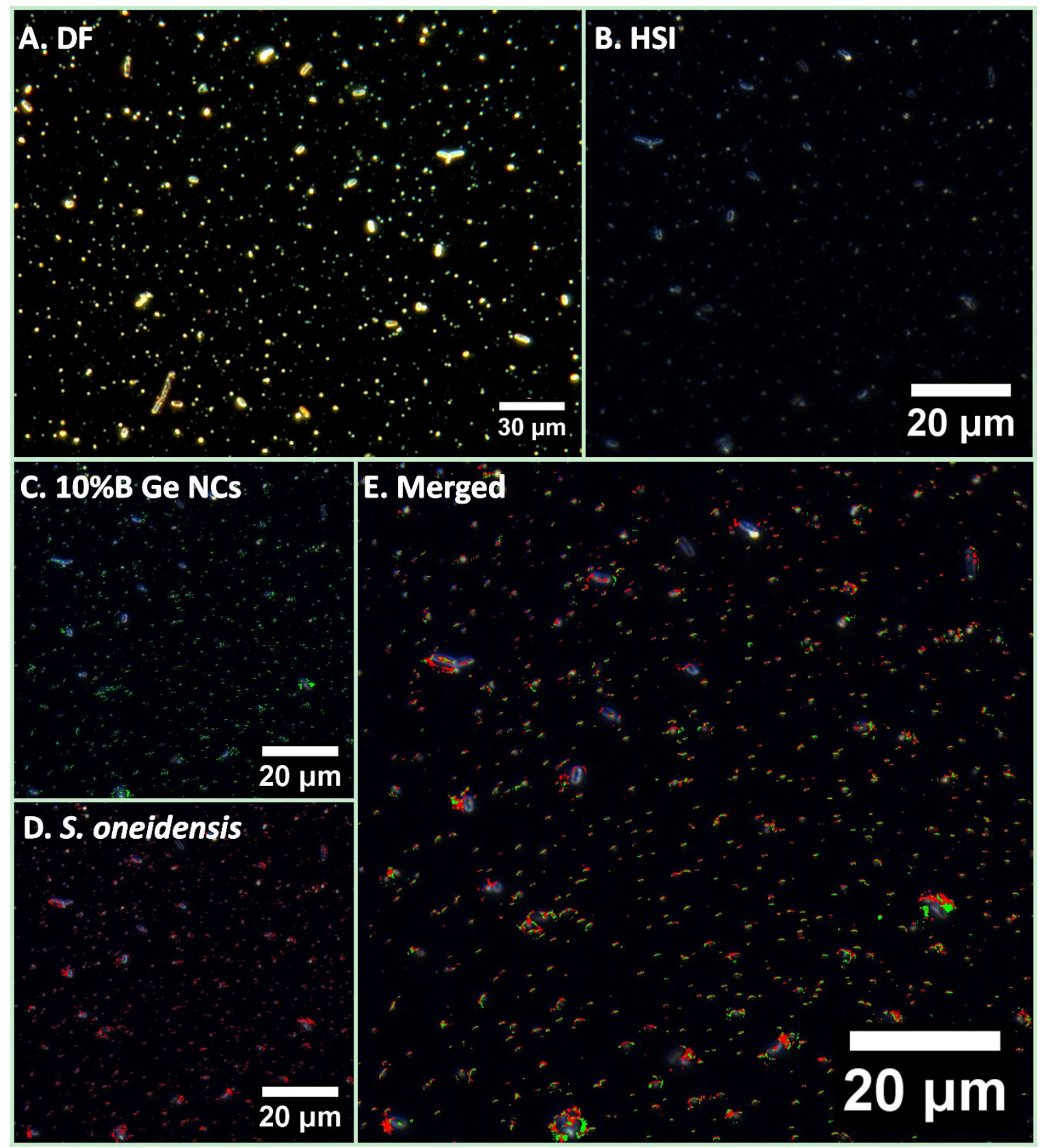

Figure S22. Cytoviva enhanced dark-field analysis of the association between 10\% B-doped Ge NCs with S. oneidensis. (A) dark-field image of bacteria exposure solution, (B) hyperspectral image of bacteria exposure solution. Mapping (C) 10\% B-doped Ge NCs alone and (D) bacteria cells alone in the hyperspectral data. (E) The merge of mapping results $(C)$ and $(D)(10 \%$ Bdoped Ge NCs are false-colored with green while bacteria cells with red). 


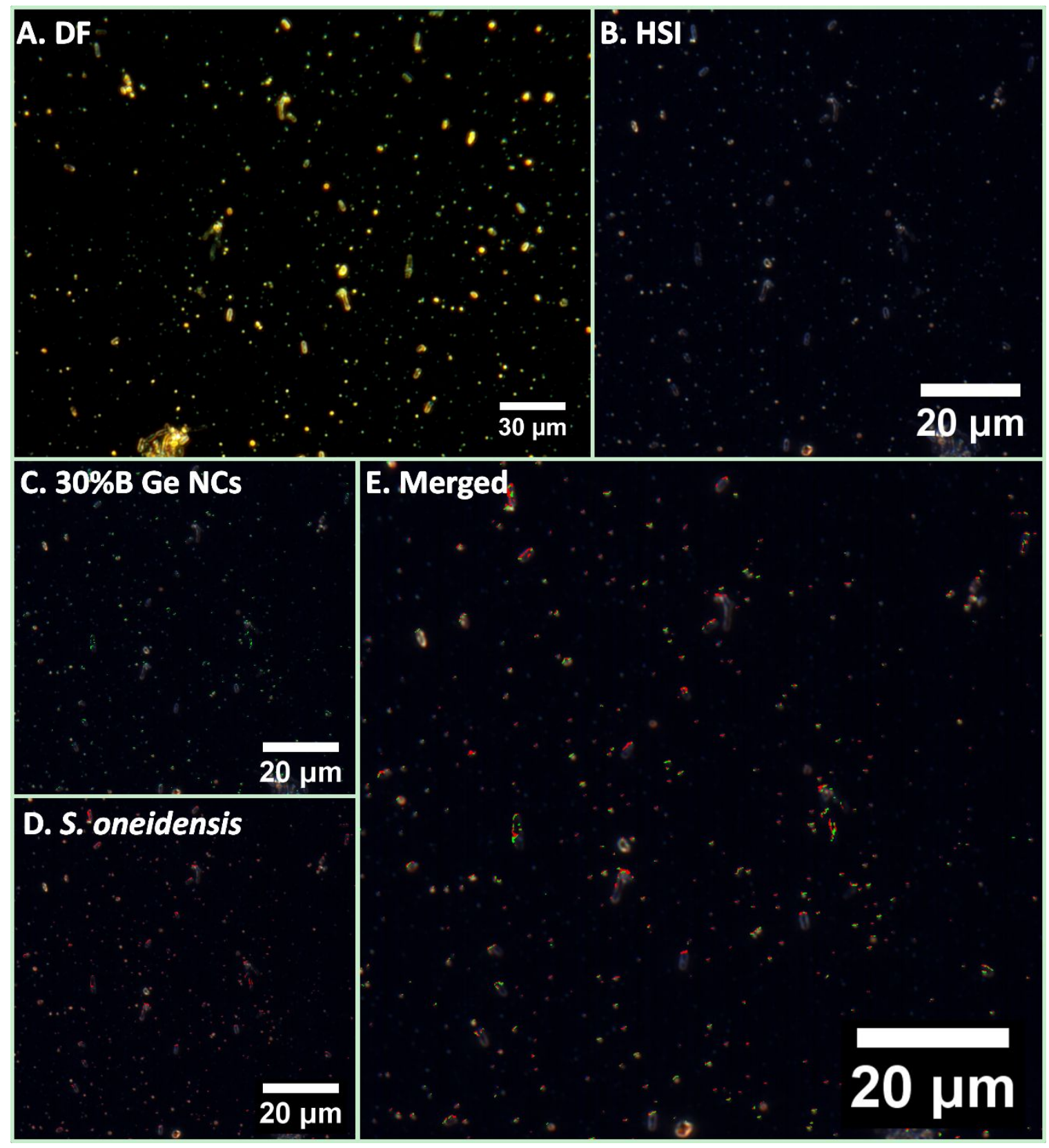

Figure S23. Cytoviva enhanced dark-field analysis of the association between $30 \%$ B-doped Ge NCs with $S$. oneidensis. (A) dark-field image of bacteria exposure solution, (B) hyperspectral image of bacteria exposure solution. Mapping (C) 30\% B-doped Ge NCs alone and (D) bacteria cells alone in the hyperspectral data. (E) The merge of mapping results (C) and (D) $(30 \% \mathrm{~B}-$ doped Ge NCs are false-colored with green while bacteria cells with red). 


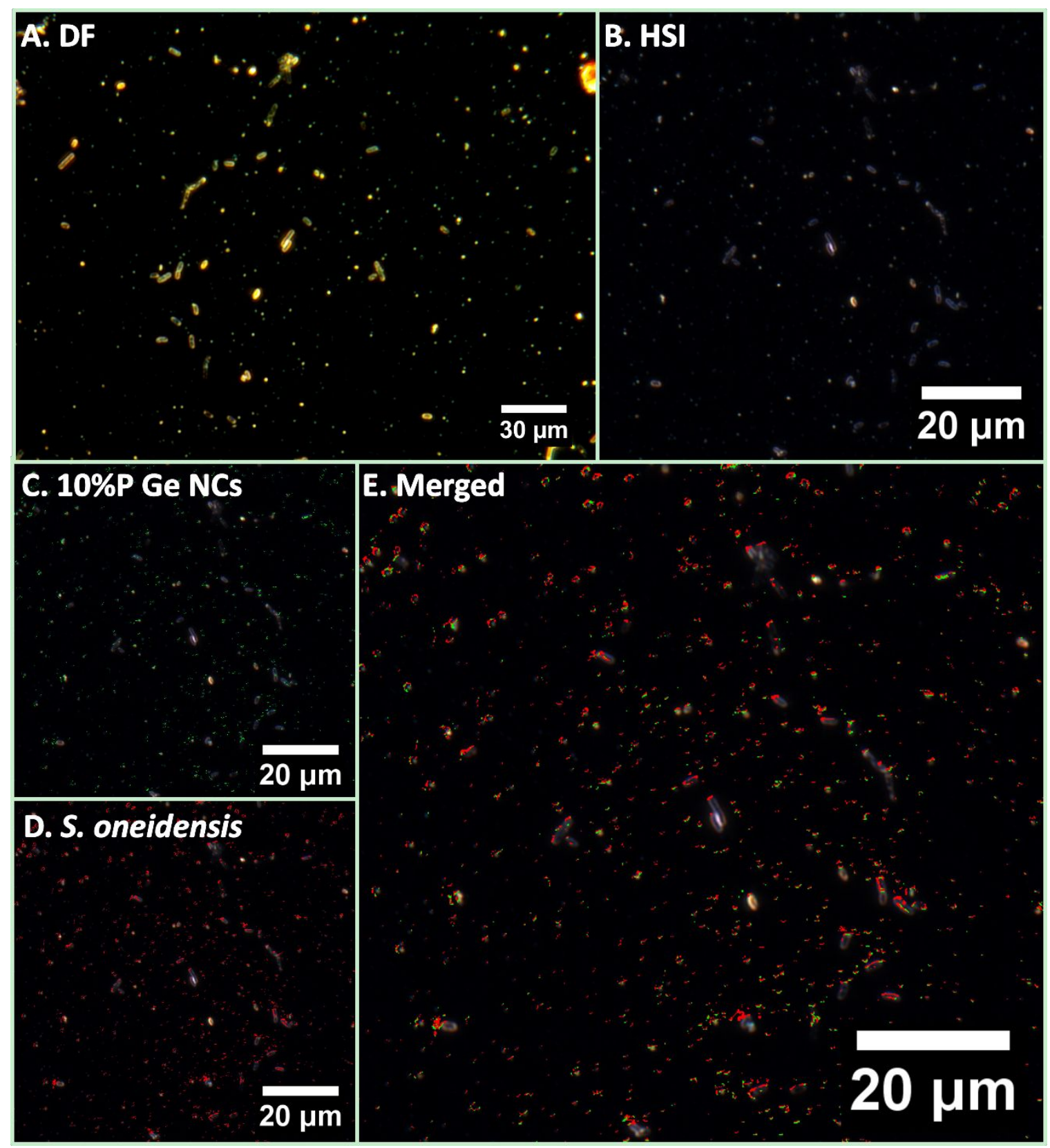

Figure S24. Cytoviva enhanced dark-field analysis of the association between $10 \% \mathrm{P}$-doped $\mathrm{Ge}$ NCs with $S$. oneidensis. (A) dark-field image of bacteria exposure solution, (B) hyperspectral image of bacteria exposure solution. Mapping (C) 10\% P-doped Ge NCs alone and (D) bacteria cells alone in the hyperspectral data. (E) The merge of mapping results (C) and (D) (10\% P-doped Ge NCs are false-colored with green while bacteria cells with red). 


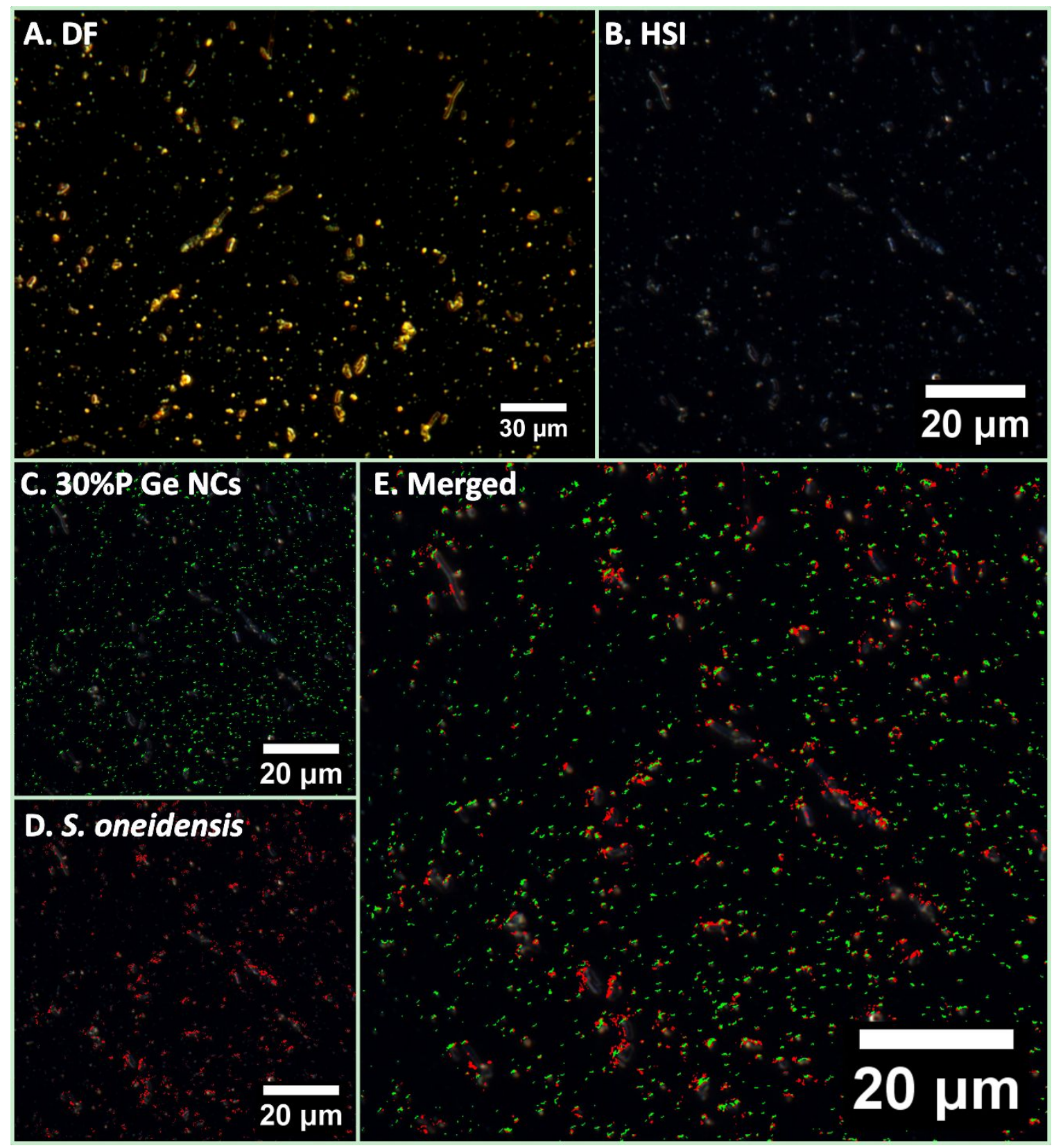

Figure S25. Cytoviva enhanced dark-field analysis of the association between 30\% P-doped Ge NCs with $S$. oneidensis. (A) dark-field image of bacteria exposure solution, (B) hyperspectral image of bacteria exposure solution. Mapping (C) 30\% P-doped Ge NCs alone and (D) bacteria cells alone in the hyperspectral data. (E) The merge of mapping results (C) and (D) (30\% P-doped Ge NCs are false-colored with green while bacteria cells with red). 


\section{A. S. oneidensis}
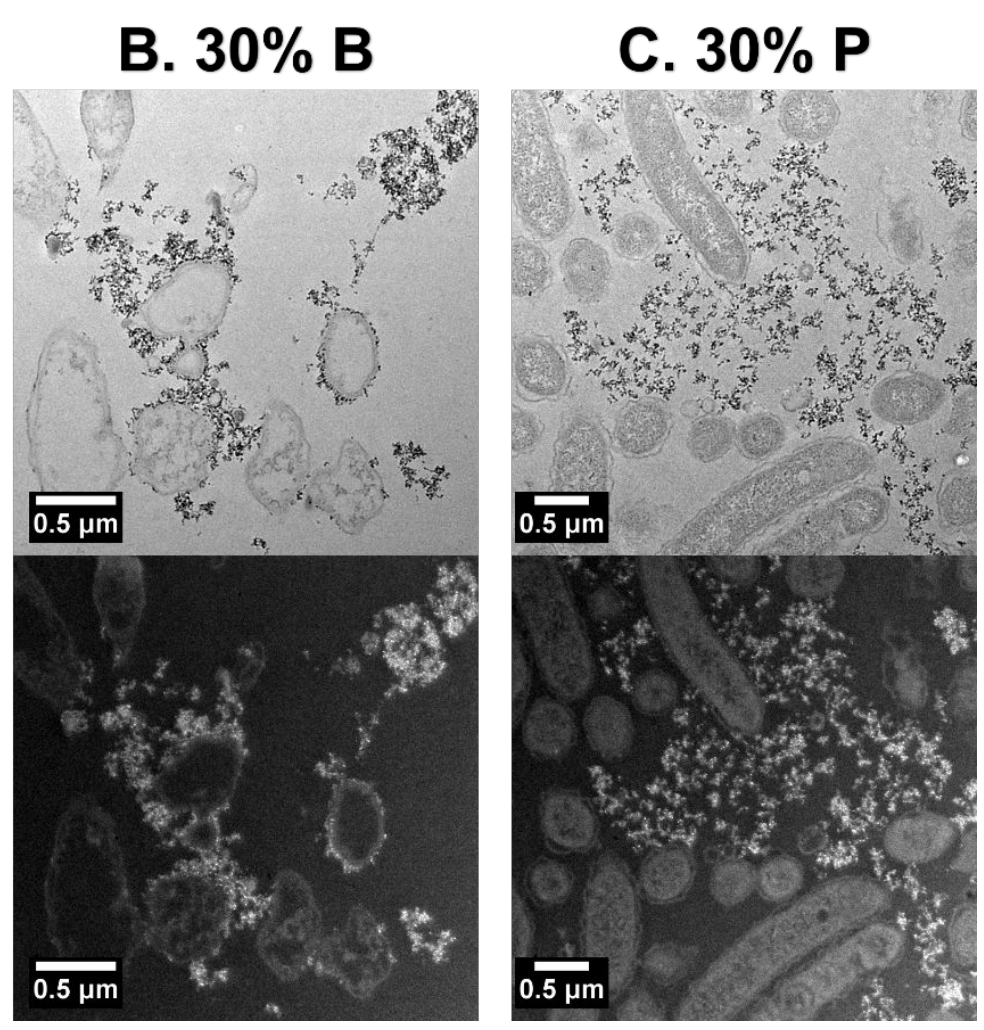

Figure S26. (upper level) Bright-field TEM images for (A) S. oneidensis cells and bacteria cells exposed to (B) 30\% B-doped Ge NCs and (C) 30\% P-doped Ge NCs; (lower level) Dark-field TEM images for bacteria cells exposed to (B) 30\% B-doped Ge NCs and (C) 30\% P-doped Ge NCs. 